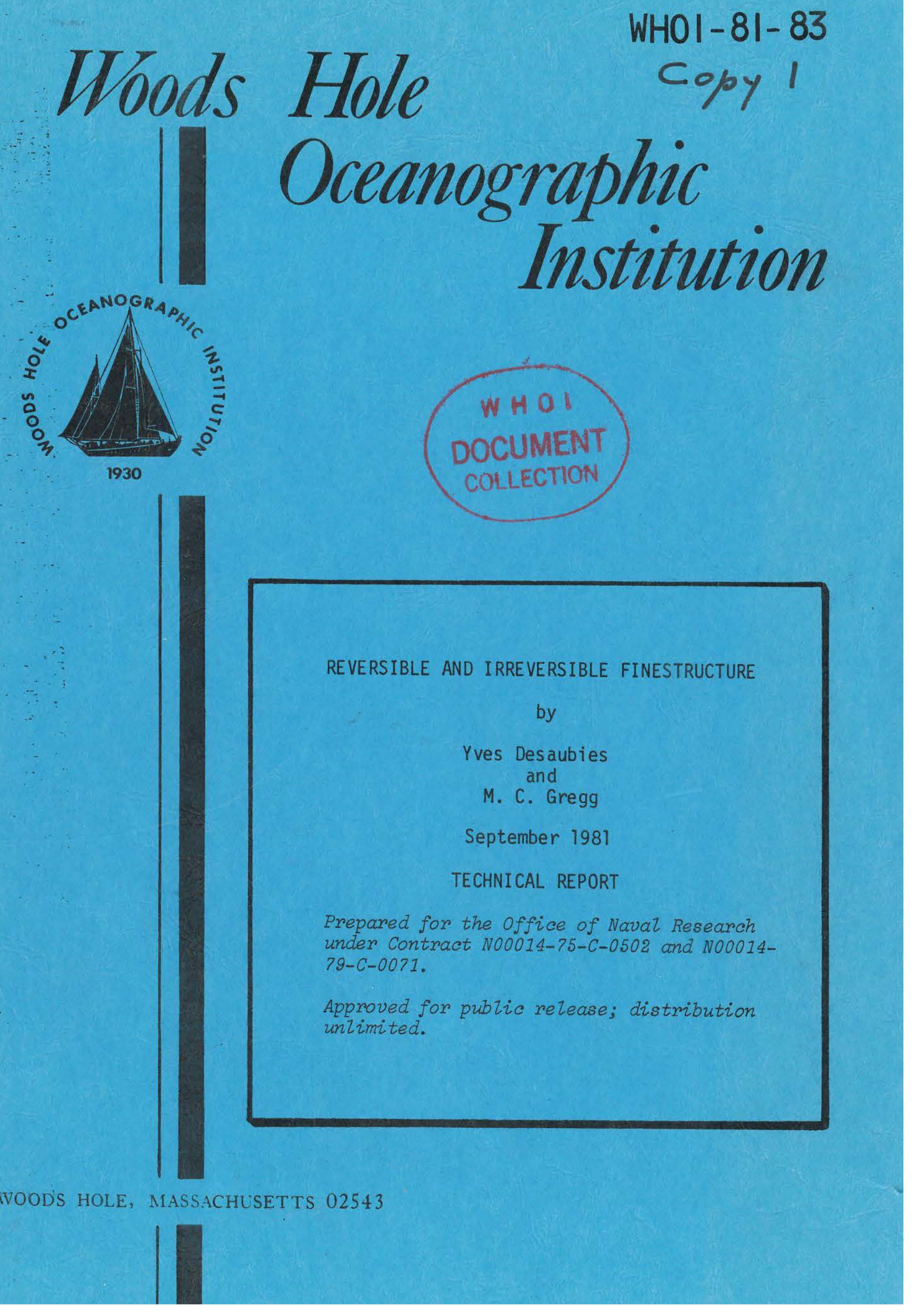




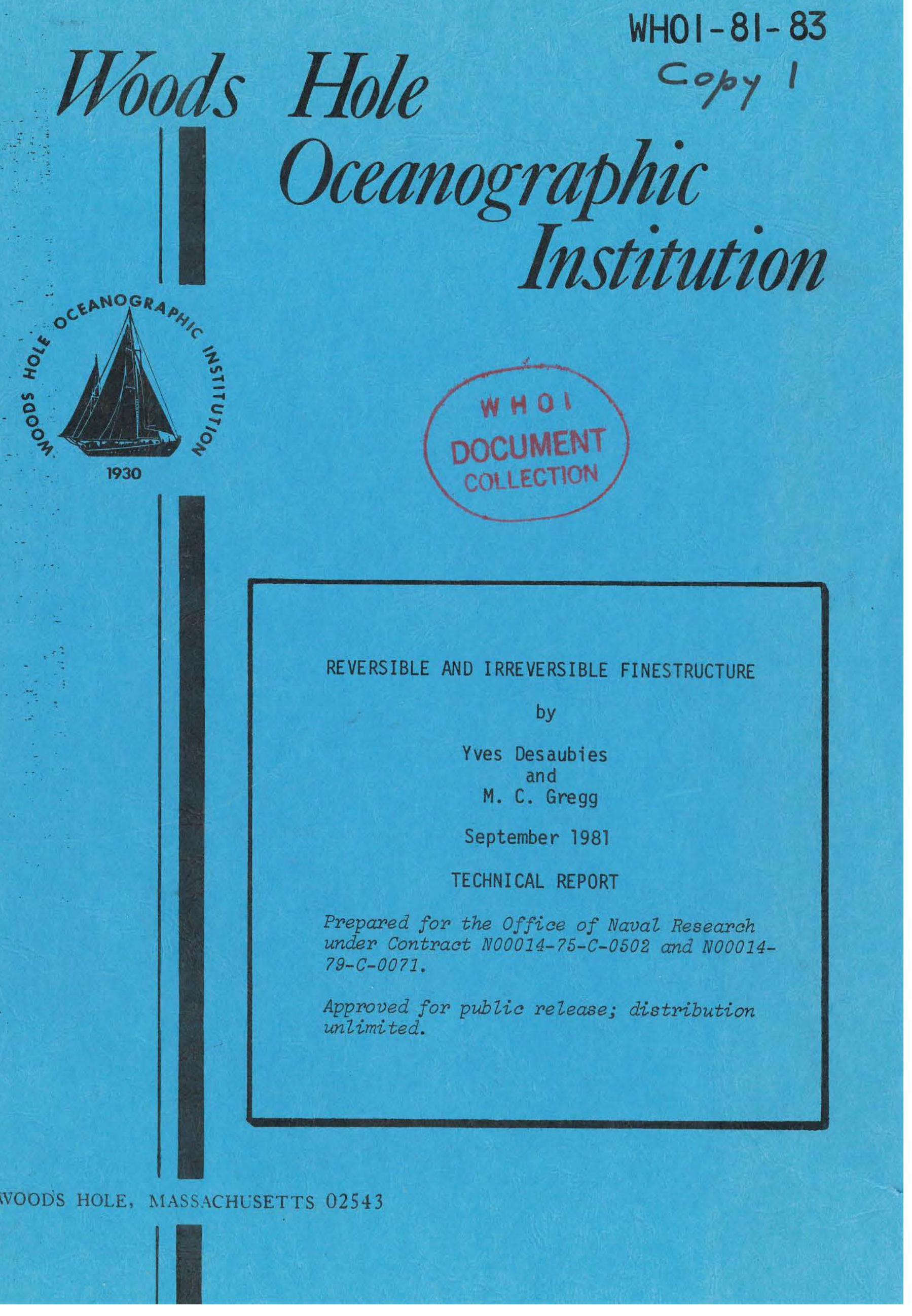


WHOI -81-83

\title{
REVERSIBLE AND IRREVERSIBLE FINESTRUCTURE
}

by

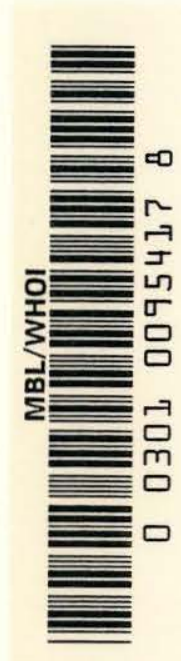
Yves Desaubies
and

M. C. Gregg

WOODS HOLE OCEANOGRAPHIC INSTITUTION

Woods Hole, Massachusetts 02543

\author{
September 1981
}

\section{TECHNICAL REPORT}

Prepared for the office of lvaval Research under Contracts N00014-75-C-0502 and N00014-79-C-0071.

Reproduction in whole or in part is permitted for any purpose of the United States Govermment. In citing this report in a bibliography, the reference given should be to: Journal of Physical Oceanography 11(4): 541-546 (April 1981).

Approved for public release; distribution unlimited.

Approved for Distribution: Earl E. Hays, Chairman

Department of Ocean Engineering 

Reprinted from Journal of Physical Oceanography, Vol. 11, No. 4, April 1981
American Meteorological Society
Printed in U. S. A.

\title{
Reversible and Irreversible Finestructure
}

\author{
Yves Desaubies \\ Woods Hole Oceanographic Institution, Woods Hole, MA 02543 \\ M. C. GREGG \\ Applied Physics Laboratory, Department of Oceanography, University of Washington, Seattle 98195
}

(Manuscript received 14 September 1979, in final form 29 December 1980)

\section{ABSTRACT}

\begin{abstract}
Various statistics of temperature profiles are examined in an attempt to distinguish irreversible structures due to mixing from reversible distortions induced by internal wave straining. Even if all the low gradient regions were the result of mixing events, an analysis of the profiles shows that such events are rare and most often incomplete. An upper bound on the mixing effectiveness is obtained; it increases as the vertical scale decreases. Taking next the opposite view that internal wave straining is the sole process, an analytic model is developed to calculate the probability density function of temperature gradients. The model considers the straining by a weakly nonlinear Gaussian internal wave field of a linear temperature profile. The nonlinearity of the field is essential to account for the skewness of the probability distributions. Comparisons with data are quite satisfactory at scales larger than $\sim 2 \mathrm{~m}$, less so at smaller scales. We conclude that nonlinear effects are important; at scales larger than $\sim 2 \mathrm{~m}$ straining is dominant with very little mixing, while at smaller scales irreversible structures are more prevalent.
\end{abstract}

\section{Introduction}

The presence of finestructure has been noted by all observers who have made continuous profiles in lakes or the oceans. Variability in even single profiles is ubiquitous and can be seen most clearly by displaying the temperature gradient (Fig. 1). If the gradient is formed by first-differencing the profile, it is found that the variability increases as the differencing interval is decreased until the diffusive cutoff is reached at scales of a few $\mathrm{cm}$. The term finestructure is usually applied to fluctuations with scales of $\sim 1 \mathrm{~m}$ and greater, which are too great to be directly affected by molecular diffusion.

Investigators have ascribed the origin of the finestructure to several processes. Based on gradient fluctuations found in bathythermograph traces, Eckart (1948) pointed out the importance of deformation of the temperature field by velocity shear. Because much of the shear is due to internal waves, these fluctuations can be termed reversible finestructure since they result in no mixing. More recently, Cooper and Stommel (1968) emphasized the irreversible aspects of finestructure that is created when a mixing event alters the profile.

Distinguishing between these two aspects of finestructure is important for developing a better understanding of internal waves and mixing. Much of the information about internal waves has been obtained by assuming that the temperature field is a passive tracer of water motion. In particular, the spectral parameterization of Garrett and Munk (1975) was developed by assuming that fluctuations in the temperature field are linearly related to the displacement field. Any irreversible structures thus constitute an undesirable noise in the observations. The opposite situation occurs when it is assumed that the temperature field represents only the vestiges of past mixing events. If internal wave deformation were not important, low gradient sections would be the sites of the most recent events, and high gradients would mark their boundaries.

An additional impetus for understanding the nature of finestructure comes from acoustics. Recent works (Desaubies, 1978; Ewart, 1980; Flatté et al., 1981) have identified finestructure as an important factor in producing amplitude fluctuations in the horizontal transmission of high-frequency signals.

In reviewing the literature about finestructure, we found that some authors have developed simple analytical models which assume that the temperature profile contains thin, completely mixed layers. In these "sheet and layer" models, all of the temperature change occurs across the sheets. Although the statistics of $\Delta T / \delta$ ( $\delta$ is the differencing interval) exhibit a good fit to data for $\delta$ larger than the scales of either sheets or layers, it has never 


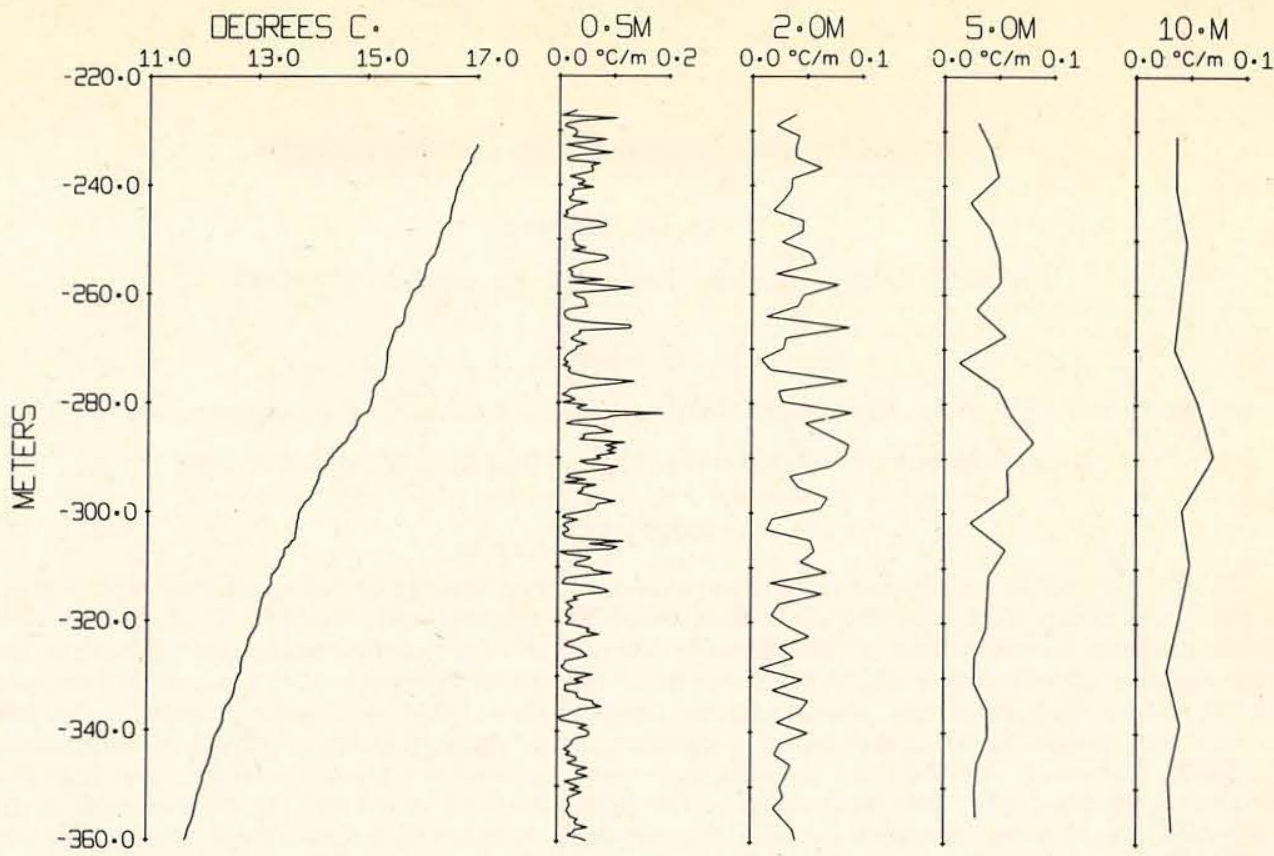

FIG. 1. Deconvolved MSR profile from the main thermocline of the central North Pacific. The spatial resolution of the record is $\sim 1 \mathrm{~cm}$. Plots to the right are temperature gradients $(\Delta T / \delta)$ formed by first-differencing the temperature over the $\delta$ scales indicated. As $\delta$ is decreased the variability in $\Delta T / \delta$ decreases as does the average separation of local maxima.

been demonstrated that the model is physically correct. In contrast, other authors have asserted that wave deformation is the dominant effect. From the high gradients apparent in Fig. 1, it is evident that such a mechanism requires very high strain over scales as small as $1 / 2 \mathrm{~m}$. The consistency of such strain with the prevailing statistical description of the wave field (Garrett and Munk, 1975; henceforth referred to as GM 75) also has not been presented.

In this paper we describe our investigations of both the irreversible and reversible aspects of finestructure. In considering irreversible finestructure, we used high-resolution temperature profiles to determine whether the small-scale structure is in fact consistent with the sheet and layer model. Following this approach we have used the data to put bounds on the effectiveness of the mixing. In considering the effects of internal wave strain, we began with the heat equation and considered the importance of both linear and nonlinear terms. To obtain quantitative estimates of the deformation for comparison with the $\Delta T / \delta$ data, a simple model, which is implicitly nonlinear, was developed.

\section{Irreversible finestructure}

\section{a. Sheets, layers and Poisson processes}

In a series of flow visualization studies, Woods (1968) observed the deformation of dye markers injected into the seasonal thermocline near Malta. One set of experiments used the distortion of wakes left by dropped dye pellets to examine vertical shear. Based on the observed patterns, Woods concluded that "the (seasonal) thermocline is a series of thin laminar-flow sheets of high static stability separated by weakly turbulent layers of only moderate density gradient and a few meters thick". Subsequent dye packets released into the sheets spread laterally, revealing waves and occasional shear instabilities along the sheets.

The qualitative model of the thermocline described by Woods was made the basis for quantitative models by subsequent investigators. Fundamental to the sheet and layer model is the assumption that finestructure is created by discrete mixing events, which occur randomly in space and time. Therefore, sections of profile with lower-thanaverage gradients are the sites of the most recent events, while higher-than-average gradients mark the boundaries between mixed sections. Implicit in the model is the assumption of a clear distinction between sheets and layers. In the same vein, McKean (1974) treated the temperature profile, which was assumed to be equivalent to density, as a Poisson process in which the vertical thickness of mixing events is a random variable. The Poisson model is illustrated schematically in Fig. 2. Since the sheets, with thickness $L_{s}$, are assumed to be much thinner than the differencing interval $\delta$, the 
gradients appear as impulse functions. Thus, the variability in $\Delta T / \delta$ is due to differing numbers of sheets within the successive intervals used to form $\Delta T / \delta$.

Hayes et al. (1975) used the Poisson probability distribution as the basis for an analytic model of the probability density function $p(\Delta T / \delta)$. Their treatment assumed that most of the sheets and layers had vertical scales less than the resolution of the CTD data. Therefore, they used Poisson statistics to predict the number of temperature jumps within intervals of $\delta=1-10 \mathrm{~m}$. Following this approach, the probability of obtaining $n$ sheets in $\delta$ is $p(n)$ $=(\mu \delta)^{n} e^{-\mu \delta} / n$ !, where $\mu$ is the average number of sheets per meter. It was further assumed that the layers are completely mixed, i.e., with zero gradient, and that the temperature jump across each sheet is proportional to the length of the preceeding layer. From this a gamma probability distribution was obtained for $p(\Delta T / \delta)$. It was found that $\mu=1.6 \mathrm{~m}^{-1}$ resulted in a good fit of the analytic distribution to the data. A somewhat more elaborate approach was followed by Joyce and Desaubies (1977), who computed $\mu$ from the profiles. In this case, the assumption was implicitly made that the minimum differencing interval, $\delta=0.3 \mathrm{~m}$, was not much greater than the thickness of the sheets; hence the separation of successive maxima gave the layer thicknesses (shown schematically in Fig. 3). The $\mu$ values ranged from 0.43 to $2 \mathrm{~m}^{-1}$ as the estimated noise in the data was varied from 0 to $2 \mathrm{~m}{ }^{\circ} \mathrm{C}$. For $\delta \sim L_{s}$ much of the variability in $\Delta T / \delta$ results from the random positioning of the differencing intervals with respect to the sheets.

Although the analytic curves developed from these models exhibited good agreement with the

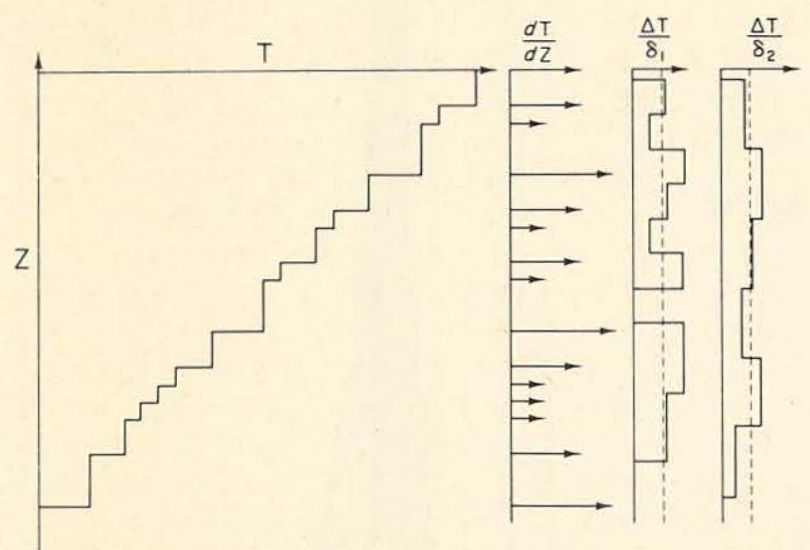

FIG. 2. Schematic view of the Poisson model of the thermocline. The temperature profile is assumed to be composed of discrete homogeneous sections (layers) separated by thin regions of high gradient (sheets). The sheets are assumed to be thinner than the differencing intervals of interest. Therefore, the variability in $\Delta T / \delta$ records that use $\delta \leqslant L_{s}$ is due to the different numbers of sheets that occur in successive $\delta$ intervals.
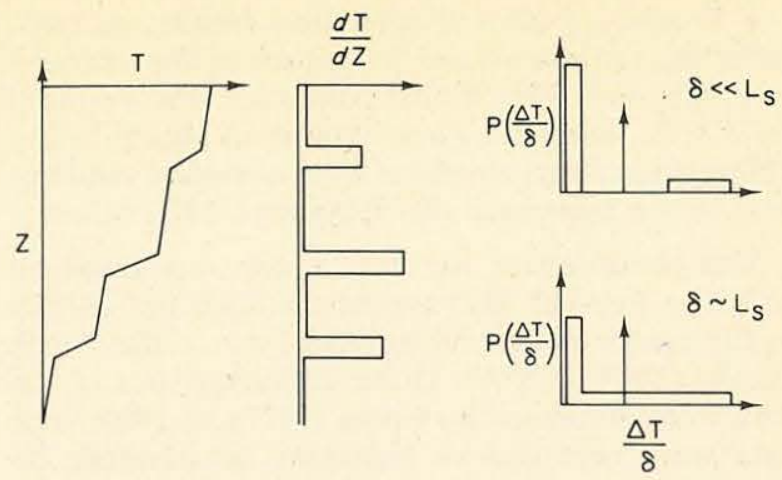

FIG. 3. Schematic view of the thermocline in which sheets have thicknesses $\geqslant \delta$. In the ideal case with $\delta \ll L_{s}$, the pdf would be bimodal. For $\delta \approx L_{s}$ even if the actual sheets had only one gradient value, the $\Delta T / \delta$ values would have a wide range due to the random positioning of the differencing intervals with respect to the sheets.

data, particularly with $p(\Delta T / \delta)$ for $\delta \geqslant 1 \mathrm{~m}$, there has been no demonstration that the representation of the temperature profile as a Poisson process is correct. Poisson statistics are applicable in situations where there are distinct and definable objects or events (Cox and Lewis, 1966); e.g., the arrival of electrons in a phototube, the scattering of acoustic energy from individual members of a school of fish, or the distribution of galaxies in space. The events may be complex, with random amplitudes and finite duration, but a unique definition must exist. The range of $\delta$ and $\mu$ values used in the sheet and layer models is equivalent to assuming that layer thicknesses are in the microstructure range, which is too small to be resolved by conventional CTD measurements. To investigate whether the temperature profile is in fact a Poisson process (i.e., whether distinct sheet and layer structures exist at scales of centimeters to tens of centimeters) we have used temperature data that resolved structures to the diffusive cut-off.

\section{b. Examination of high-resolution data}

If the assumptions made by previous investigators are correct, then as $\delta$ is decreased, a distinct separation of $\Delta T / \delta$ levels found in sheets versus layers should emerge as $\delta$ becomes equal to and then smaller than $L_{s}$. The probability density functions (pdf) in Fig. 3 illustrate this schematically. Thus, if there are definable sheets and layers we should expect for $\delta<L_{s}$ that:

- $p(\Delta T / \delta)$ should show a large number of very low gradients and a small number of high gradients. In an extreme case $p(\Delta T / \delta)$ might be bimodal.

- By defining a "trigger level" of $\Delta T / \delta$ it should be possible to distinguish sheets from layers in a first differenced profile. The location of the sheets should be stable as the magnitude of $\delta$ is varied. 
- Profiles of $\Delta T / \delta$ should show trends, or runs, reflecting the non-stationary nature of the temperature structure. This should contrast to the situation for $\delta \gg L_{s}$, when the large number of sheets in any differencing interval will result in complete randomness in the sequential distribution of $\Delta T / \delta$ values.

The observations we have used were obtained with the free-fall Microstructure Recorder (MSR) in the center of the subtropical gyre of the North Pacific $\left(28^{\circ} \mathrm{N}, 155^{\circ} \mathrm{W}\right)$. Other characteristics of the data were discussed by Gregg (1977a,b; 1980). The data were recorded as high-pass temperature by filtering and amplifying the signal across a thermistor. During analysis, the profiles were reconstructed by digitally deconvolving the data to remove the effect of the electronic transfer function. A separate lowgain, but more stable, temperature record was used to correct for drift in the high-gain channel. The rms noise of the recorded data was $8 \mu^{\circ} \mathrm{C}$. Because the analysis was performed with extended precision on a large computer, the noise was not increased.

The pdf's in Fig. 4 were obtained from five profiles taken in the depth range of $200-400 \mathrm{~m}$. Differencing intervals from 0.1 to $10 \mathrm{~m}$ were used (a larger number of STD profiles was used to form the $10 \mathrm{~m}$ values). For large $\delta$ the distributions tend to a Gaussian form, reflecting the fact the $\Delta T$ values result from many apparently independent small fluctuations. As $\delta$ decreases, the distributions become very skewed with a mode (most probable
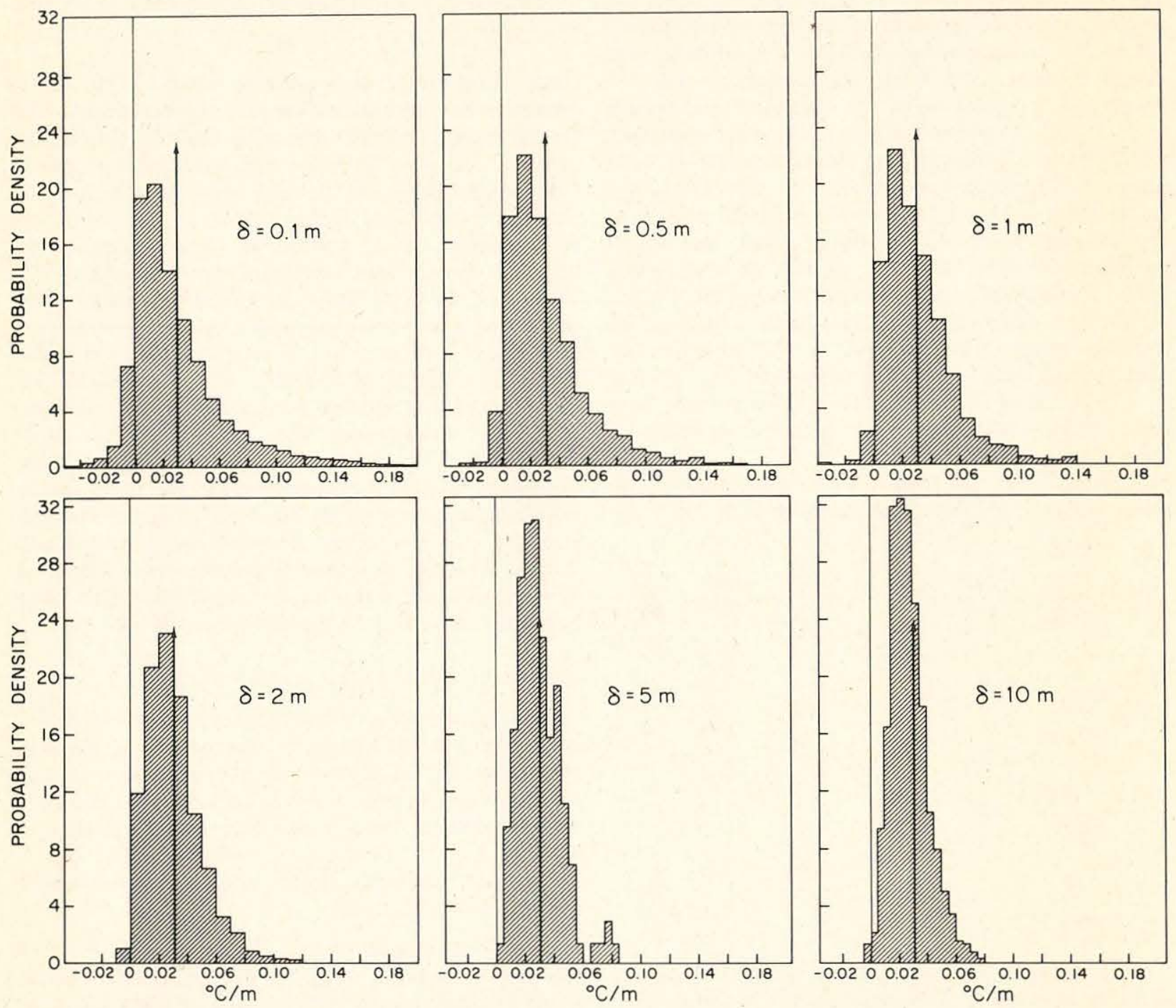

FIG. 4. Probability density functions $p(\Delta T / \delta)$ for $\delta$ values ranging from 0.1 to $10 \mathrm{~m}$. Even with $\delta=0.1 \mathrm{~m}$, the pdf does not tend toward the distribution expected for a sheet and layer thermocline. The pdf's are continuous rather than bimodal and do not provide a basis for an objective classification of low gradients versus high gradients. 
value) of about one-half the mean gradient and a tail extending to high gradients.

The curve in Fig. 4 for $\delta=0.1 \mathrm{~m}$ is not appreciably different than that for $\delta=1.0 \mathrm{~m}$, and definitely does not show either of the forms portrayed schematically in Fig. 3. The same is true, if even smaller $\delta$ values are used. [For example, refer to Fig. 4 in Gregg (1980) where $\delta$ $=1.5 \mathrm{~mm}$ was used.] From pdf's such as these there is no basis for choosing a trigger value to separate sheets from layers. As a comparison, one of the few cases we have found of a sheet and layer profile was shown in Fig. 5a of Gregg and Sanford (1980). The pdf of that record, which was formed with $\delta=0.8 \mathrm{~m}$, resembled the example in Fig. 4 for $\delta \approx L_{s}$.

Although it is apparent that there is no clear distinction between sheets and layers, we wondered whether nevertheless it might not be possible to define typical scales of the high and low gradient sections by using the approach of Joyce and Desaubies. In doing so, we followed their definition of $\mu^{-1}$ to be the average separation of successive maxima in the first-differenced records. When applied to the section shown in Fig. 1, values of $0.032,0.048,0.15$ and $0.48 \mathrm{~m}^{-1}$ were obtained as $\delta$ decreased from 10 to $0.5 \mathrm{~m}$. In each case the mean spacing of the maxima corresponds to $(3-4) \delta$ and is thus a characteristic of the differencing interval rather than the temperature field. As evident in
Fig. 5, examination of records at smaller scales reveals a similar situation. A few structures on the $0.25 \mathrm{~m}$ scale persist as local maxima to the scale of the diffusive cutoff (e.g., the sharp gradient near $314.5 \mathrm{~m}$ in Fig. 5) but most are resolved into $\mathrm{cm}$ scale maxima and minima.

In spite of the lack of distinct sheets and layers, inspection of records such as Fig. 5 leaves one with the impression that there are structures $\sim 1 / 4$ to $2 \mathrm{~m}$ thick. For example, note that the shape of the $0.25 \mathrm{~m}$ difference record provides a good envelope for the maximum amplitudes of the smaller scale gradients.

One way of objectively testing this impression is the run test, a simple non-parametric procedure which determines whether a data series is completely random or whether trends exist (Bendat and Piersol, 1971). The test consists of counting the number of times data values cross the median value for the series; consecutive data points on the same side of the median are termed runs. For a stationary, or homogenous, series of $N$ points, the expected number of runs $R$ is $N / 2$. Values of $R$ beyond the confidence limits about $N / 2$ show that the series is not completely random, but contains non-random structures, or trends.

Five first-differenced MSR records with $\delta$ values of 0.5 to $10 \mathrm{~m}$, including the one shown in Fig. 1, were examined. As seen in Fig. 6, the results are consistent with the $\Delta T / \delta$ values being independent random variables for $\delta>2 \mathrm{~m}$, but not for $\delta$ of $1 \mathrm{~m}$ or
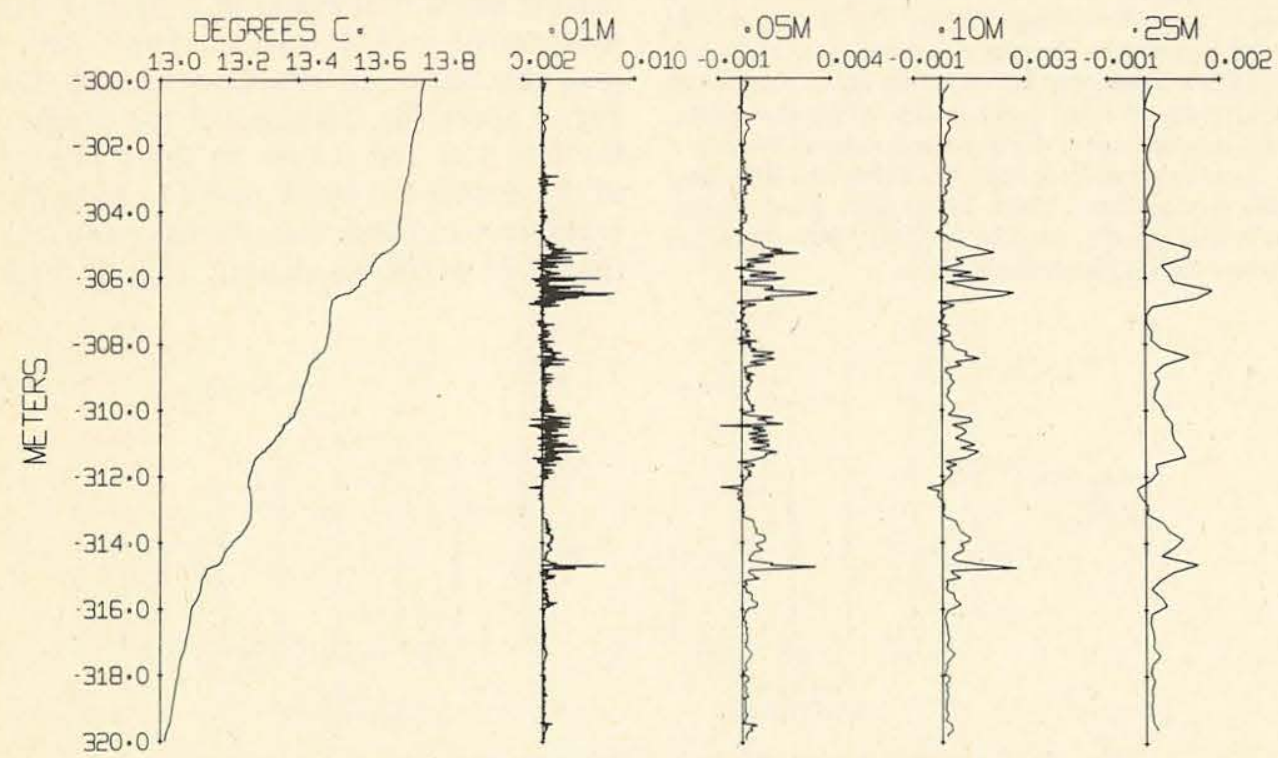

Fig. 5. Expanded plot of a $20 \mathrm{~m}$ thick section of the data displayed in Fig. 1. Differencing intervals of $\delta=0.01$ to $0.25 \mathrm{~m}$ have been used. The separation of local maxima continues to decrease as $\delta$ is decreased to the differsive cutoff at $\delta=0.01 \mathrm{~m}$. Since these data had low microstructure levels, the increase in variability at small scales is the least that can be expected. Although some features persist as well-defined high gradients, e.g., the spike near $-315 \mathrm{~m}$, many are resolved into clusters of large amplitude gradients a few centimeters thick. 


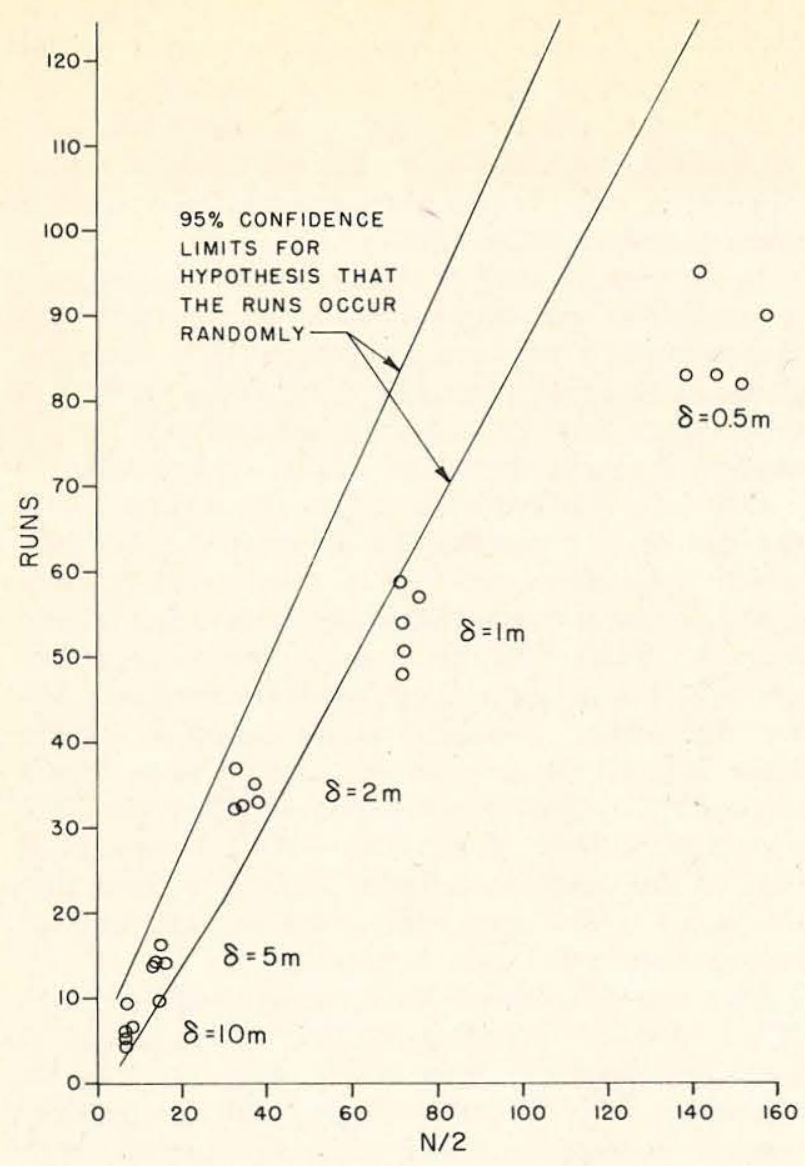

FIG. 6. Run tests applied to five first-differenced MSR records from $200-400 \mathrm{~m}$ in the central North Pacific. The tests were run for five differencing intervals. The plot gives the number of runs, defined as consecutive values on the same side of the median, as a function of $N / 2$, where $N$ is the number of points in the record. For a completely random series the expected number of runs is $N / 2$. With $\delta<2 \mathrm{~m}$ the number of runs lies below the $95 \%$ confidence limits for random data. Thus, larger scale gradient features occur in a completely random manner, but there are coherent structures with scales less than $2 \mathrm{~m}$. less. Thus, temperature differences over scales of $2 \mathrm{~m}$ and larger occur sequentially in a random manner, while thinner ones tend to be clustered.

\section{c. Limits on mixing}

From the examination discussed above, we have concluded that there is no objective basis for the sheet and layer model of the typical thermocline nor for the use of Poisson statistics. The gradient fluctuations are much too irregular to permit an objective separation of the profile into high versus low gradient sections. This, however, does make a strong statement about mixing in the thermocline: individual mixing events do not often go to completion, and rarely produce homogeneous sections of profile. Rather, they produce relatively minor changes in the stratification.

If a profile that initially has the mean density gradient $\bar{g}$ is completely mixed over a thickness $h$, the increase in potential energy (PE) is $G \bar{g} h^{3} / 12$. This is the maximum increase that can occur. If the mixing ceases when the gradient has been reduced to $g$, with $0<g<\bar{g}$, then the ratio of the actual increase in $\mathrm{PE}$ to the maximum possible is:

$$
\frac{\Delta \mathrm{PE}}{(\Delta \mathrm{PE})_{\max }}=\frac{\bar{g}-g}{\bar{g}} .
$$

In Gregg (1980) a demonstration was made to show that mixing patches occur randomly with respect to the finestructure. Thus, the typical mixing event will begin in a section with an initial gradient of $\bar{g}$. The final gradient, with $g<\bar{g}$, will give the degree of mixing achieved. The graphs in Fig. 7 show the fractions of the profile with $g<\bar{g}$ for $\delta=5 \mathrm{~m}$ and $0.5 \mathrm{~m}$. In the $5 \mathrm{~m}$ case only $14 \%$ of the profile has been mixed to more than $1 / 2$ of the maximum and less than $1 \%$ has been mixed to more than 0.85 of the maximum. The corresponding plot
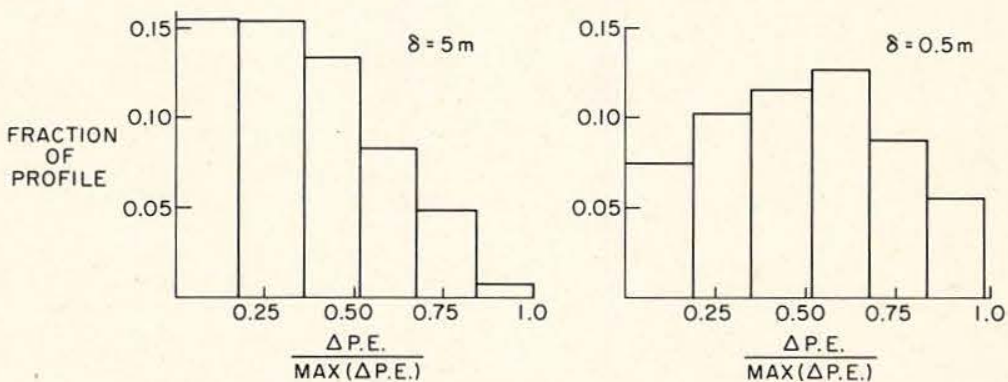

FIG. 7. Histograms showing fraction of the profile having temperature gradient less than the mean over vertical separation between 5 and $0.5 \mathrm{~m}$. The abscissas are the ratio of the increase in potential energy to that corresponding to complete mixing. Thus a value of 0.75 corresponds to a gradient that is $1 / 4$ of the mean and hence was mixed to $3 / 4$ of completion, if it was formed irreversibly. Since reversible strain can be partly responsible for the gradient levels, these histograms represent upper limits on the irreversible increase in potential energy. 
for $0.5 \mathrm{~m}$ is much higher at the greater completion levels, showing that events are much more likely to mix strongly at the smaller scales.

The fractions of the profile given in Fig. 7 are based on the assumption that all of the gradient fluctuations are due to mixing and thus give upper bounds on the degree to which the profile has actually been mixed. These bounds should be considered in estimates of heat fluxes due to breaking internal waves, which usually assume that if an event occurs it will mix to completion. For example, Garrett (1979) points out that if the vertical shear spectrum cuts off at $0.1 \mathrm{cpm}$ then most of the shear instabilities will occur over scales of about $12 \mathrm{~m}$. Assuming that all events mix to completion, he estimated that one event every $30 \mathrm{~h}$ would be required to produce a turbulent heat flux equivalent to $K_{z} \sim 10^{-4} \mathrm{~m}^{2} \mathrm{~s}^{-1}$. From plots similar to Fig. 7, it is evident that only a few percent of the events actually result in such complete mixing and that most produce less than $1 / 2$ of the maximum possible change in PE. Thus, assuming the extreme case that all finestructure is irreversible, the frequency of occurrence should be increased by a factor of $2-4$ to produce the same heat flux. This, in turn, implies that $12 \mathrm{~m}$ scale overturning events should be more common in profile data. Using Garrett's values, one would expect 5 to 10 active events in a $1 \mathrm{~km}$ profile. As discussed by Gregg (1980) the data have not exhibited such frequent large-scale events.

\section{The role of nonlinear straining on temperature observations}

In the absence of diffusion, which takes place over time scales large compared to internal wave periods, the heat equation can be written

$$
\partial_{t} T+\mathbf{u} \cdot \nabla T=0 .
$$

The temperature field at point $\mathbf{s}$ can be expressed as the sum of a time independent part, representing the mean state, and a fluctuating component due to the internal waves:

$$
T(s, t)=\bar{T}(s)+T^{\prime}(s, t),
$$

whereby (1) becomes

$$
\partial_{t} T^{\prime}(s, t)+\mathbf{u} \cdot \nabla \bar{T}+\mathbf{u} \cdot \nabla T^{\prime}=0 .
$$

In the oceanic internal wave field $\mathbf{u}$ and $T$ are such that $\mathbf{u}_{H} \cdot \nabla \bar{T} \ll w \partial_{z} \bar{T}$, so that $\mathbf{u} \cdot \nabla \bar{T} \simeq w \partial_{z} \bar{T}$. If (3) is linearized it then takes the usual form

$$
\partial_{t} T^{\prime}+w \partial_{z} \bar{T}=0
$$

or

$$
T^{\prime}=-\eta \partial_{z} \bar{T},
$$

where we have introduced the vertical displacement

$$
\eta=\int^{t} w d t .
$$

Within these approximations the temperature fluctuation and its rate of change at one point are simply proportional to the internal wave vertical displacement and velocity, respectively.

The relation (5) can be derived in a slightly different, but illuminating way. We assume now that if there were no internal waves, the temperature profile would be the equilibrium profile $T_{0}(z)$; in the presence of a vertical displacement field $\eta(z, t)$ the temperature observed at some depth $z_{*}$, $T\left(z_{*}, t\right)$, is given by

$$
T\left(z_{*}, t\right)=T_{0}\left[z_{*}-\eta\left(z^{\prime}, t\right)\right],
$$

where $z^{\prime}$ is defined implicitly by

$$
z^{\prime}(t)+\eta\left(z^{\prime}, t\right)=z_{*} .
$$

(For fixed observation depth $z_{*}$ the depth of origin of the isotherm $z^{\prime}$ is a function of time). The field $\eta(z)$ can be expanded as

$$
\eta\left(z_{*}\right)=\eta\left(z^{\prime}\right)+\left.\eta\left(z^{\prime}\right) \frac{\partial \eta}{\partial z}\right|_{z^{\prime}}+\cdots,
$$

and approximated by $\eta\left(z^{\prime}\right) \simeq \eta\left(z_{*}\right)$ provided $|\partial \eta / \partial z|$ $\ll 1$. Then the temperature can be expressed in the form

$T\left(z_{*}, t\right) \simeq T_{0}\left[z_{*}-\eta\left(z_{*}, t\right)\right] \simeq T_{0}\left(z_{*}\right)-\eta\left(z_{*}, t\right) \frac{\partial T_{0}}{\partial z}$

provided that $T_{0}(z)$ is smooth enough. The result (9) is identical to (5). To derive (9) two independent assumptions have been made, 1) the internal wave field is linear with negligible strain: $|\partial \eta / \partial z| \ll 1$; 2 ) the equilibrium profile $T_{0}(z)$ is a slowly varying function of depth, at least over lengths of the order of the displacement.

If the first hypothesis were valid but not the second, i.e., if there were in $T_{0}(z)$ sharp gradients (and large curvature) presumably related to the presence of irreversible structures with time scales longer than the internal wave period, then the expansion (9) is incomplete and should include higher order terms:

$$
T=T_{0}-\eta \frac{\partial T_{0}}{\partial z}+\frac{1}{2} \eta^{2} \frac{\partial^{2} T_{0}}{\partial z^{2}} \cdots .
$$

Taking the time average we obtain for the mean and fluctuating parts:

$$
\begin{aligned}
\bar{T} & =T_{0}+\frac{1}{2} \overline{\eta^{2}} \frac{\partial^{2} T_{0}}{\partial z^{2}}+\cdots, \\
T^{\prime} & =-\eta \frac{\partial T_{0}}{\partial z}+\frac{1}{2}\left(\eta^{2}-\overline{\eta^{2}}\right) \frac{\partial^{2} T_{0}}{\partial z^{2}}-\cdots .
\end{aligned}
$$


Thus if irreversible structures with high gradients which persist over the averaging time are present in the profile $T_{0}(z)$, we conclude that:

- The relations (5) and (9) do not hold and there is no simple relation between observed temperature fluctuations and internal wave displacements.

- The nonlinear terms in (3) are important because $\nabla \bar{T} \simeq \nabla T^{\prime}$.

- The time-averaged temperature at a point is not equal to $T_{0}: \bar{T} \neq T_{0}$ [Eq. (11)].

The difficulties associated with this situation constitute the so-called fine structure contamination problem and have been the object of a number of phenomenologic studies (Reid, 1971; Phillips, 1971; Garrett and Munk, 1971; McKean, 1974; Joyce and Desaubies, 1977; Eriksen, 1978; Levine, 1979). We shall not discuss it further here.

Another interesting situation arises when the nonlinearity of the wave field cannot be neglected. Indeed one can estimate (see below) that the rms strain $\left[\overline{[\partial \eta / \partial z)^{2}}\right]^{1 / 2} \simeq \mathrm{O}(1)$ in the ocean. To demonstrate the resulting effects we shall now assume that $T_{0}(z)$ is very smooth and write $T_{0}(z)=\bar{g} z$, i.e., a linear profile with constant mean gradient $\bar{g}$.

If the expression (5), now written $T^{\prime}=-\bar{g} \eta$ is substituted in the nonlinear term of (3) we have

$$
\partial_{t} T^{\prime}+\left(1-\partial_{z} \eta\right) w \bar{g}-\bar{g} \mathbf{u}_{H} \cdot \nabla \eta=0,
$$

showing that the nonlinear vertical advective term $w \partial_{z} T^{\prime}$ is of order $\partial_{z} \eta$ compared to the linear term. The horizontal advection term is of the same order, as can be seen by comparing $w \partial_{z} \eta$ to $\mathbf{u}_{H} \cdot \nabla \eta$, or more simply by substituting a Fourier component $T^{\prime} \sim \exp (i \mathbf{k} \cdot \mathbf{x})$ into $\mathbf{u} \cdot \nabla T^{\prime}$ :

$$
\mathbf{u} \cdot \nabla T^{\prime} \sim i \mathbf{k}_{H} \cdot \mathbf{u}+i k_{v} w .
$$

For an incompressible fluid, $\boldsymbol{\nabla} \cdot \mathbf{u}=0$, so that for a single wave component the two terms cancel exactly; for a superposition of waves we expect the two terms to be of comparable magnitude.

We see then that the nonlinear terms, which represent the advection of the waves by themselves, are proportional to the strain $\partial_{z} \eta$ and to the slope $\nabla_{H} \eta$. Eq. (13) shows that the temperature fluctuations are no longer proportional to the displacement field.

This last remark can be clarified by considering Eq. (7), which becomes, for a linear profile $T_{0}=\bar{g} z$,

$$
T\left(z_{*}, t\right)-\bar{g} z_{*}=-\bar{g} \eta\left(z^{\prime}, t\right) .
$$

The temperature fluctuation is proportional to the displacement $\eta\left(z^{\prime}, t\right)$, not to $\eta\left(z_{*}\right)$. The latter is the displacement undergone by the isotherm whose mean depth $z_{*}$ is the observation depth; at $z_{*}$, however, at any given time, another isotherm is observed, which has mean position $z^{\prime}$ and has been displaced by $\eta\left(z^{\prime}, t\right)$ to reach $z_{*}$. As time elapses different isotherms are observed at $z_{*}$ and $z^{\prime}$ is a function of time. The difference between $\eta\left(z_{*}\right)$ and $\eta\left(z^{\prime}\right)$ is given by (8) and is proportional to the strain $\partial \eta / \partial z$. It is important to specify clearly how the displacement is defined when it is used to derive a vertical velocity.

Our goal is now to consider the implications of these considerations for the relationship between the displacement and temperature statistics.

\section{A model of the temperature statistics}

The fundamental description of a random process is given by its probability density function. We propose, therefore, to derive here an expression for the probability density of temperature, assuming that we know the statistics of the displacement field. The problem we addressed can be stated as follows. Consider a temperature field $T_{0}(z)$ to be function of depth only, and let this field be deformed by a three-dimensional, time-dependent displacement field with given probability distribution. What is the resulting probability distribution of the temperature field?

The temperature gradient $T_{0}=\bar{g} z$ is assumed constant. The displacement field is assumed to be a Gaussian process, i.e., if two particles with mean position $s_{1}$ and $s_{2}$ are displaced to $s_{1}+\xi_{1}$, and $s_{2}$ $+\xi_{2}$, then the joint probability density function of $\xi_{1}, \xi_{2}$ is given by

$$
\begin{aligned}
& p\left(\xi_{1}, \xi_{2} \mid \mathbf{d}\right) \\
& =(2 \pi)^{-6}|\mathbf{C}|^{-1 / 2} \exp \left[\frac{-1}{2|\mathbf{C}|} \sum_{i, j=1}^{6}\left|C_{i j}\right| \zeta_{i} \zeta_{j}\right],
\end{aligned}
$$

where $\zeta$ is the 6-vector $\left(\xi_{1}, \xi_{2}\right), \mathbf{C}$ is the covariance matrix with elements $\left\langle\boldsymbol{\xi}_{i}, \boldsymbol{\xi}_{j}\right\rangle, \mathbf{C}$ its determinant, and $\left|C_{i j}\right|$ the cofactor of $\left\langle\boldsymbol{\xi}_{i}, \boldsymbol{\xi}_{j}\right\rangle$. The covariances are functions of $\mathbf{d} \equiv \mathbf{s}_{1}-\mathbf{s}_{2}$, and this fact is emphasized in the notation $p\left(\xi_{1}, \xi_{2} \mid \mathbf{d}\right)$. The Gaussianity of the vertical displacement has been checked by Briscoe (1977) and Gregg (1977a) on the basis of temperature data. They concluded that their data were consistent with a normal distribution. However, because of the subtleties involved in defining the displacement, their results must be viewed with caution. There is some theoretical justification for assuming a normal distribution if the internal wave field consists of a set of randomly phased weakly interacting waves. Then the central limit theorem predicts a normal distribution. However if the interactions between various wave components are strong, as suggested by Holloway (1980), definite phase relations are established and the central limit theorem does not apply. Nonetheless, we shall use the distribution (16) as our starting point; if it could be shown that another expression were more ap- 
propriate, our formalism would remain entirely applicable.

To render the problem tractable we must make a more drastic approximation. It can be seen that the relation in (15) involves only the vertical component $\eta$ of the displacement $\xi$; we shall therefore neglect all horizontal displacements so that a particle observed at some point is assumed to have originated somewhere on a vertical passing through that point. In the context of the preceding section this is tantamount to neglecting the horizontal advective terms in the heat equation. It follows from this simplification that our final results have only a qualitative value which will be discussed in due time.

The distribution (16) becomes now

$$
\begin{aligned}
p\left(\eta_{1}, \eta_{2} \mid y\right) & =\left[2 \pi Z^{2}\left(1-\rho^{2}\right)\right]^{-1 / 2} \\
& \times \exp \left[-\frac{\eta_{1}{ }^{2}+\eta_{2}{ }^{2}-\rho(y) \eta_{1} \eta_{2}}{2 Z^{2}\left(1-\rho^{2}\right)}\right],
\end{aligned}
$$

where $y=\left|z_{2}{ }^{\prime}-z_{1}{ }^{\prime}\right|$ is the mean vertical separation of the isotherms considered, $\rho=\rho(y)=\left\langle\eta_{1} \eta_{2}\right\rangle /$ $Z^{2}$ is the displacement correlation, $Z^{2}=\left\langle\eta_{1}{ }^{2}\right\rangle$ $=\left\langle\eta_{2}{ }^{2}\right\rangle$ its variance.

\section{a. Temperature statistics at one sensor}

Consider first a simple problem in which a sensor at a fixed depth $z_{*}$ measures temperature. Given the displacement distribution (17) we wish to determine the probability density function of observed temperature. To render the problem more interest- ing we assume, in this example only, that the variance $Z^{2}$ is a function of depth, i.e., $Z=Z(z)$.

From Eq. (7) the observed temperature is

$$
\left.\begin{array}{c}
T=T\left(z_{*}\right)=T_{0}\left[z_{*}-\eta\left(z^{\prime}\right)\right] \\
=\bar{g}\left[z_{*}-\eta\left(z^{\prime}\right)\right]=\bar{g} z^{\prime} \\
z^{\prime}+\eta\left(z^{\prime}\right)=z_{*}
\end{array}\right\} .
$$

Clearly, since $\eta$ is a random variable, so is $z^{\prime}$. From Eq. (17) an isotherm with mean depth $z^{\prime}$ has displacements distributed according to

$$
\begin{aligned}
p\left(\eta \mid z^{\prime}\right)=\left[(2 \pi)^{1 / 2} Z\right]^{-1} & \\
& \times \exp \left[-\frac{\eta^{2}}{2 Z^{2}\left(z^{\prime}\right)}\right] \equiv f\left(\eta, z^{\prime}\right) .
\end{aligned}
$$

The cumulative distribution $F(\hat{T})$ is defined as the probability that a given observation $\hat{T} \leqslant T$. For the event $\hat{T} \leqslant T$ to happen it is necessary and sufficient that the isotherm $T$ (with mean depth $\bar{z}=T / \bar{g}$ ) be displaced to a level above the observation depth $z_{*}: \eta(\bar{z}) \geqslant z_{*}-\bar{z}$. This is expressed by

$$
\begin{aligned}
F(T) & =\operatorname{Pr}(\hat{T} \leqslant T)=\operatorname{Pr}\left[z_{*}-\eta(\bar{z}) \leqslant T / \bar{g}\right] \\
& =\operatorname{Pr}\left[\eta(\bar{z}) \geqslant z_{*}-T / \bar{g}\right]=\operatorname{Pr}\left[\eta(\bar{z}) \geqslant z_{*}-\bar{z}\right] \\
& =\int_{z_{*}-\bar{z}}^{\infty} f(\eta, \bar{z}) d \eta \\
& =(2 \pi)^{1 / 2} \int_{z_{*}-\bar{z}}^{\infty} Z^{-1} \exp \left(-\eta^{2} / 2 Z^{2}\right) d \eta, \quad(20)
\end{aligned}
$$

wherein $Z=Z(\bar{z})=Z(T / \bar{g})$ and $\operatorname{Pr}[\quad]$ is the probability of the event in brackets. The density function is

$$
\begin{aligned}
p\left(T \mid z_{*}\right)= & \partial_{T} F(T)=\bar{g}^{-1} \partial_{\bar{z}} F=\left[(2 \pi)^{1 / 2} \bar{g}\right]^{-1} Z^{-1} \exp \left[-\left(z_{*}-\bar{z}\right)^{2} / 2 Z^{2}\right]+\left(Z^{\prime} / Z^{4}\right) \int_{z_{*}-\bar{z}}^{\infty} d \eta e^{-\eta^{2} / 2 Z^{2}}\left(\eta^{2}-Z^{2}\right) \\
= & {\left[(2 \pi)^{1 / 2} \bar{g}\right]^{-1}\left[Z+\left(z_{*}-\bar{z}\right) Z^{\prime}\right] Z^{-2} \exp \left[-\left(z_{*}-\bar{z}\right)^{2} / 2 Z^{2}\right] } \\
& =-\left[(2 \pi)^{1 / 2} \bar{g}\right]^{-1} \frac{d}{d \bar{z}}\left[\frac{\left(z_{*}-\bar{z}\right)}{Z}\right] \exp \left[-\left(z_{*}-\bar{z}\right)^{2} / 2 Z^{2}\right],
\end{aligned}
$$

where, again, $Z=Z(T / \bar{g}), Z^{\prime}=d Z / d \bar{z}$.

This distribution is very different from normal; it is easy to verify that $\int p\left(T / z_{*}\right) d T=1$, as required. One also finds that

$$
\langle T\rangle \neq \bar{g} z_{*}=T_{0}\left(z_{*}\right) .
$$

In words, the mean temperature observed at some depth $z_{*}$ is different from the temperature of the isotherm which has that depth as its equilibrium position. This is a consequence of our temporary assumption that the displacement variance $Z$ is depth dependent, and can be understood intuitively. If $Z$ increases with depth, isotherms above $z_{*}$ have smaller variance than those below and are less likely to reach $z_{*}$. Thus isotherms from below will be observed more often than those from above and the temperature distribution will be skewed, with mean temperature smaller than $T_{0}\left(z_{*}\right)$. If $Z$ is constant, as we shall assume from now on, (21) reduces to a normal distribution with mean $\langle T\rangle=\bar{g} z_{*}=T_{0}\left(z_{*}\right)$ and variance $\left\langle T^{2}\right\rangle=(\bar{g} Z)^{2}$. We have treated this example because it contains all the features of the more relevant and complex problem to be treated now.

\section{b. Temperature gradient statistics between two sensors}

Consider two sensors, fixed in space along the same vertical, at depths $z_{1}$ and $z_{2}$, let $\delta=z_{2}-z_{1}$, 


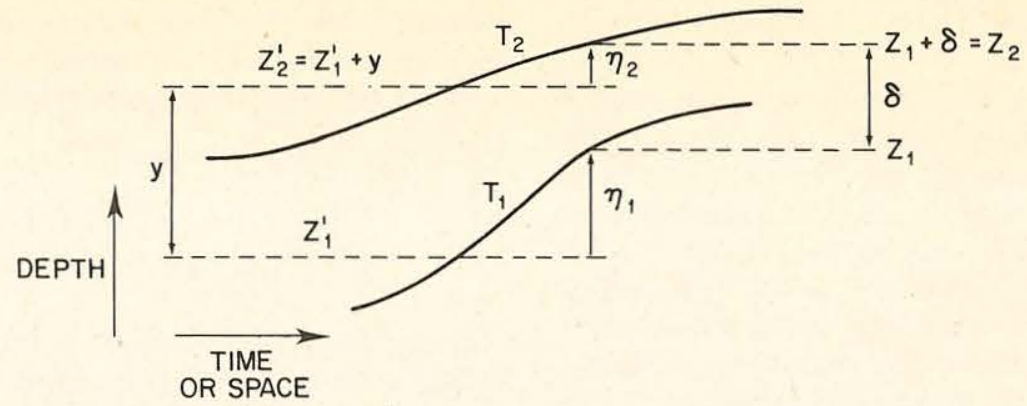

FIG. 8. Schematics of the displaced isotherms indicating the notation: an isotherm with equilibrium depth $z_{i}{ }^{\prime}$ is displaced by $\eta_{i}$ and is observed at $z_{i}=z_{i}{ }^{\prime}+\eta_{i}$.

(Fig. 8). Temperature is observed at each sensor and we wish to determine the statistics of the temperature difference $T_{2}-T_{1}$. As before, the isotherm observed at $z_{i}$ originates at $z^{\prime}{ }_{i}$ and has been displaced by $\eta_{i}=\eta\left(z^{\prime}{ }_{i}\right)$; the temperature observed at $z_{i}$ is then, as in (18), $T_{i}=T_{0}\left(z_{i}\right)=\bar{g}\left[z_{i}-\eta_{i}\left(z_{i}{ }^{\prime}\right)\right]=\bar{g} z_{i}{ }^{\prime}$, with $z_{i}{ }^{\prime}+\eta_{i}=z_{i}, i=1,2$. Generalizing (20), we write the joint cumulative distribution

$$
\begin{aligned}
F\left(T_{1}, T_{2}\right)= & \operatorname{Pr}\left(\hat{T}_{1} \leqslant T_{1} \text {, and } \hat{T}_{2} \leqslant T_{2}\right) \\
= & \operatorname{Pr}\left[\bar{g}\left(z_{1}-\eta_{1}\right) \leqslant T_{1} \text { and } \bar{g}\left(z_{2}-\eta_{2}\right) \leqslant T_{2}\right] \\
= & \operatorname{Pr}\left(\eta_{1} \geqslant z_{1}-T_{1} / \bar{g} \text { and } \eta_{2} \geqslant z_{2}-T_{2} / \bar{g}\right) \\
= & \int_{z_{1}-T_{1} / \bar{g}}^{\infty} d \eta_{1} \int_{z_{2}-T_{2} / \bar{g}}^{\infty} d \eta_{2}\left[2 \pi Z^{2}\left(1-\rho^{2}\right)^{1 / 2}\right]^{-1} \\
& \times \exp \left[-\frac{\eta_{1}{ }^{2}+\eta_{2}{ }^{2}-2 \rho \eta_{1} \eta_{2}}{2 Z^{2}\left(1-\rho^{2}\right)}\right],
\end{aligned}
$$

where, as in (17), $\rho=\rho\left(z_{2}{ }^{\prime}-z_{1}{ }^{\prime}\right)=\rho\left[\left(T_{2}-T_{1}\right) / \bar{g}\right]$ is a function of the random variables $T_{i}$.

From this expression the probability density function of the observed temperature difference $T_{2}-T_{1}$ can be calculated. The algebra is rather cumbrous and is given in the appendix. The result is

$$
\begin{aligned}
p(y \mid \delta)=(2 \pi)^{-1 / 2}\left\{\left(s^{\prime \prime}\right.\right. & \left.+\frac{\left[s+(\delta-y) s^{\prime}\right]^{2}}{s^{3}}\right), \\
& \left.\times \exp \left[-\frac{1}{2}\left(\frac{\delta-y}{s}\right)^{2}\right]\right\},
\end{aligned}
$$

where $s(y)=Z\{2[1-\rho(y)]\}^{1 / 2}$ will be recognized as the rms value of the difference in displacements of two isotherms whose equilibrium separation is $y$. Distributions of related random variables can readily be derived from (24). For instance, denoting the analytical expression $p(y \mid \delta)=f(y, \delta)$, the density of the gradient $g=\bar{g} y / \delta$ is

$$
p(g \mid \delta)=\delta \bar{g}^{-1} f(\delta g / \bar{g}, \delta) .
$$

\section{Evaluation of the model}

In order to estimate the distribution of gradients given in (24), an analytical expression for the correlation $\rho(y)$ is required. If the continuous representation of the GM 75 spectrum is used (Desaubies, 1976), the wavenumber spectrum of the displacement is

$$
\begin{gathered}
\phi(\beta)=2 \pi^{-1} \beta_{*} Z^{2}\left(\beta^{2}+\beta_{*}{ }^{2}\right)^{-1}, \\
\int_{0}^{\infty} \phi(\beta) d \beta=Z^{2} ;
\end{gathered}
$$

its cosine transform is the correlation function

$$
\rho(y)=Z^{-2} \int_{0}^{\infty} \cos \beta y \phi(\beta) d \beta=\exp \left(-\beta_{*} y\right) .
$$

Thus, two parameters describe the internal wave field; the variance $Z^{2}$, proportional to the energy level; and the bandwidth $\beta_{*}$ (which is the inverse of the correlation length).

With (27) the function $s(y)=Z[2(1-\rho)]^{1 / 2}(24)$ has the properties

$$
\begin{gathered}
s(0)=0, \quad \lim _{y \rightarrow \infty} s(y)=\sqrt{2} Z, \quad s^{\prime}(y)>0, \\
\lim _{y \rightarrow \infty} s^{\prime}=0 .
\end{gathered}
$$

Some of the properties of (24) can be deduced by integration:

$$
\begin{aligned}
\operatorname{Pr}\left(a^{\prime}<g<b^{\prime}\right) & =\int_{a^{\prime}}^{b^{\prime}} p(g \mid \delta) d g=\int_{a}^{b} f(y, \delta) d y, \quad\left\{\begin{array}{l}
a=a^{\prime} \delta / \bar{g} \\
b=b^{\prime} \delta / \bar{g}
\end{array}\right. \\
& =(2 \pi)^{-1 / 2} \int_{a}^{b}\left(s^{\prime \prime}+\left[(\delta-y) s^{\prime}+s\right]^{2} / s^{3}\right) \exp \left\{-1 / 2[(\delta-y) / s]^{2}\right\} d y .
\end{aligned}
$$


Introducing $r(y)=(\delta-y) / s, r^{\prime}=-\left[s+(\delta-s) s^{\prime}\right]$ $\times s^{-2}$ and integrating the first term by parts yields

$\operatorname{Pr}\left(a^{\prime}<g<b^{\prime}\right)$

$$
=(2 \pi)^{-1 / 2}\left\{\left[s^{\prime} e^{-r^{2} / 2}\right]_{a}^{b}-\int_{r(a)}^{r(b)} e^{-r^{2} / 2} d r\right\} .
$$

Particular cases of interest are $\operatorname{Pr}(g<0)=0$, $\operatorname{Pr}(g<\infty)=1$, as required, and

$$
\operatorname{Pr}(0<g<\bar{g})=1 / 2+(2 \pi)^{-1 / 2} s^{\prime}(\delta) .
$$

Numerical integration shows that $\langle g\rangle=\bar{g}$. Because of the exponential factor in (24) values of $y$ close to $\delta$ contribute most to the density, for large $\delta$ those values of $y$ are large and the derivatives $s^{\prime}$ and $s^{\prime \prime}$ become very small as $s(y)$ approaches a constant. The distribution (24) is thus nearly normal for large $\delta$. For small $\delta$ however, the departures from Gaussian are significant, with a large skewness.

To quantify these statements the value of the parameters $Z$ and $\beta_{*}$ must be determined. Within the framework of the GM 75 model, $Z$ and $\beta_{*}$ should be universal constants. However, variations by factors of 2 or so can be expected, depending on location. For the data discussed here, Gregg (1977a) deduces, from temperature spectra, a level [see Eq. (26)].

$$
2 \pi^{-1} \beta_{*} Z^{2}=1.2 \times 10^{-1} \mathrm{~m}^{2} \mathrm{cpm},
$$

i.e.,

$$
\beta_{*} Z^{2}=1.8810^{-1} \mathrm{~m}^{2} \mathrm{cpm}=1.18 \mathrm{~m}^{2} \mathrm{~m}^{-1} .
$$

Values of $Z$ and $\beta_{*}$ consistent with GM 75 (Desaubies, 1976) are, with a buoyancy frequency of $n=3.7$ cph, $Z=6.4 \mathrm{~m}, \beta_{*}=2.88 \times 10^{-2} \mathrm{~m}^{-1}$. The shape of the curves (24) is however very sensitive to the choice of $Z$ and $\beta_{*}$; the best fit between data and the model is shown in Fig. 9, with $Z$ and $\beta_{*}$ as indicated. It is not possible to fit the data with a single choice of $Z$ and $\beta_{*}$; the gradients estimated over $\delta=5$ and $10 \mathrm{~m}$ can be fitted with parameters closest to those determined a priori from the internal wave model.
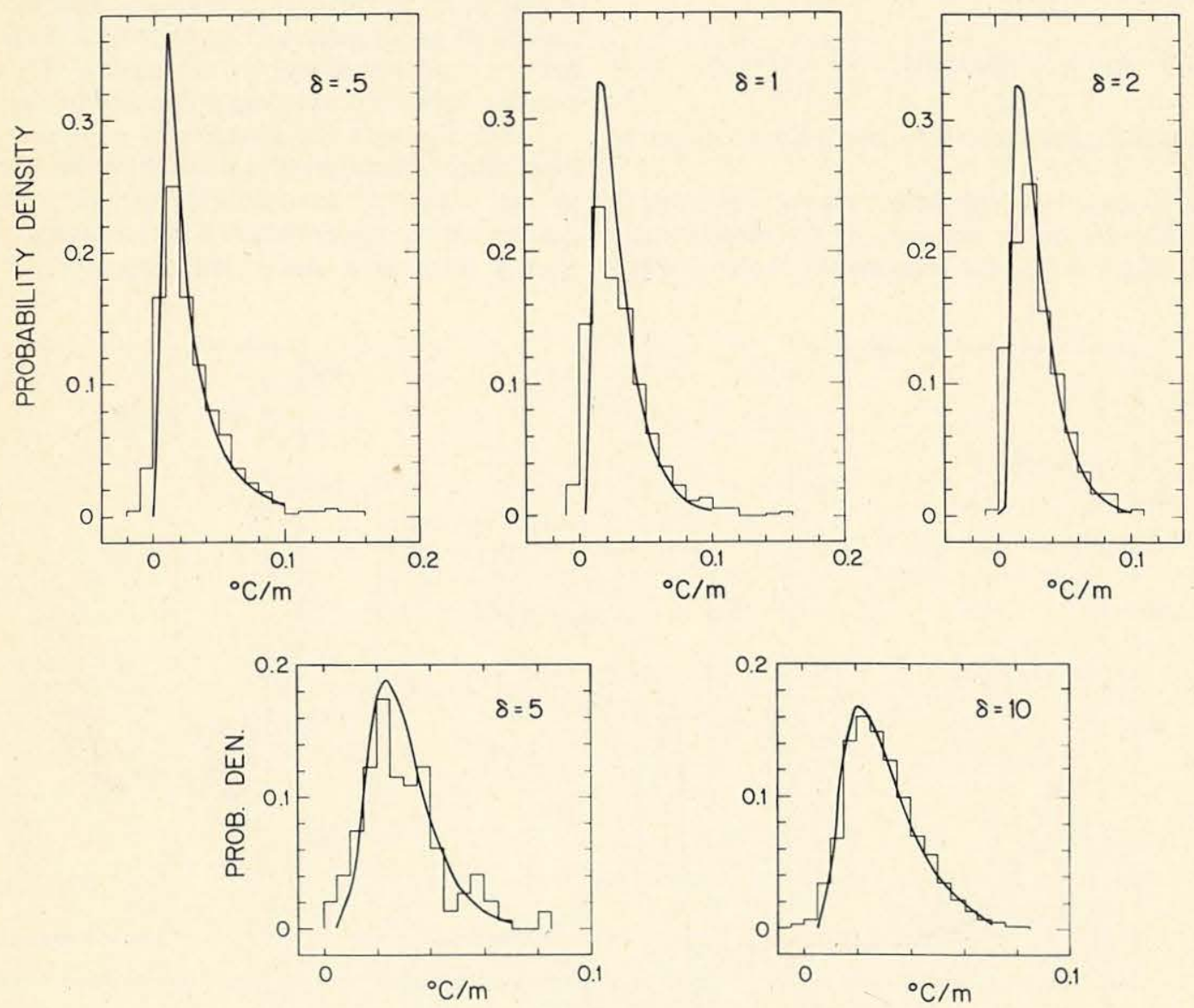

TEMPERATURE GRADIENT

Fig. 9. Comparison of the model [Eqs. (24) and (25)] with the data. Values of the parameters are given in Table 1. 
TABLE 1. Summary of the values of the parameters giving the best fit to the data (Fig. 9). The last two columns give the probability that the observed gradient is smaller than the mean.

\begin{tabular}{clccc}
\hline \hline $\begin{array}{c}\delta \\
(\mathrm{m})\end{array}$ & $\begin{array}{c}Z \\
(\mathrm{~m})\end{array}$ & $\begin{array}{c}\beta_{*} \\
\left(\mathrm{~m}^{-1}\right)\end{array}$ & $\begin{array}{c}\operatorname{Pr}(g<\bar{g}) \\
{[\text { Eq. (31)] }}\end{array}$ & $\begin{array}{c}\operatorname{Pr}(g<\bar{g}) \\
(\text { data })\end{array}$ \\
\hline 0.5 & 4 & $10^{-2}$ & 0.66 & 0.62 \\
1 & 4 & $10^{-2}$ & 0.61 & 0.59 \\
2 & 4 & $2 \times 10^{-2}$ & 0.61 & 0.57 \\
5 & 4 & $3 \times 10^{-2}$ & 0.58 & 0.58 \\
10 & 6.4 & $3 \times 10^{-2}$ & 0.58 & 0.55 \\
\hline
\end{tabular}

Nonetheless the main features of the data, i.e., the skewness with the most probable value smaller than the mean, are well reproduced with values of $Z$ and $\beta_{*}$ that are not unreasonable. The probability of observing a gradient smaller than the mean as given by (31) is shown in Table 1, together with the experimental value.

The sensitivity of the curves to the parameters is shown on Fig. 10. If the linear relation (5) was used to relate temperature to displacements there would result, in lieu of (24) and (25), a normal distribution

$$
p(y \mid \delta)=(2 \pi)^{-1 / 2} s^{-1}(\delta) \exp \left\{-\frac{1}{2}\left[\frac{\delta-y}{s(\delta)}\right]^{2}\right\} .
$$

A comparison between (24) and (32) is given in Fig. 11.

Even though our simplified model reproduces successfully the main features of the temperature gradient distributions for reasonable choices of the internal wave parameters, it is unsatisfactory that one set of parameters does not fit all the curves. We can think of four reasons for this deficiency.

First, the model considers only the vertical advection, neglecting the horizontal terms. Since we have shown the two effects to be of the same order of magnitude, this approximation is an obvious source of error. To account for horizontal advection the general expression (16) should be used instead of (17). The essential difference would be that two particles observed at $z_{2}$ and $z_{1}$ on the same vertical have, in their equilibrium position, a separation with horizontal and vertical components. The correlation of their displacements, being a function of the separation, would thus be decreased.

Second, as discussed above, irreversible processes are responsible for some of the temperature structure. It is perhaps significant in that respect that our straining model reproduces the observed histograms (Fig. 9) for the largest separations $(\delta=5$ and $10 \mathrm{~m})$ with the values of $Z$ and $\beta_{*}$ closest to those determined a priori from the internal wave field, while for the smaller separations $(\delta \leqslant 2 \mathrm{~m})$ we had to tune $Z$ and $\beta_{*}$ to fit the data. This is consistent with the results of the run tests (Fig. 6), which indicate that for $\delta<2 \mathrm{~m}$ trends occur, suggesting that at these smaller scales irreversible processes are present.

Third, the way the histograms were constructed from temperature profiles does not entirely conform to the statistical assumptions of the model. The gradient data were obtained by choosing a differencing interval $\delta$ and calculating, for successive
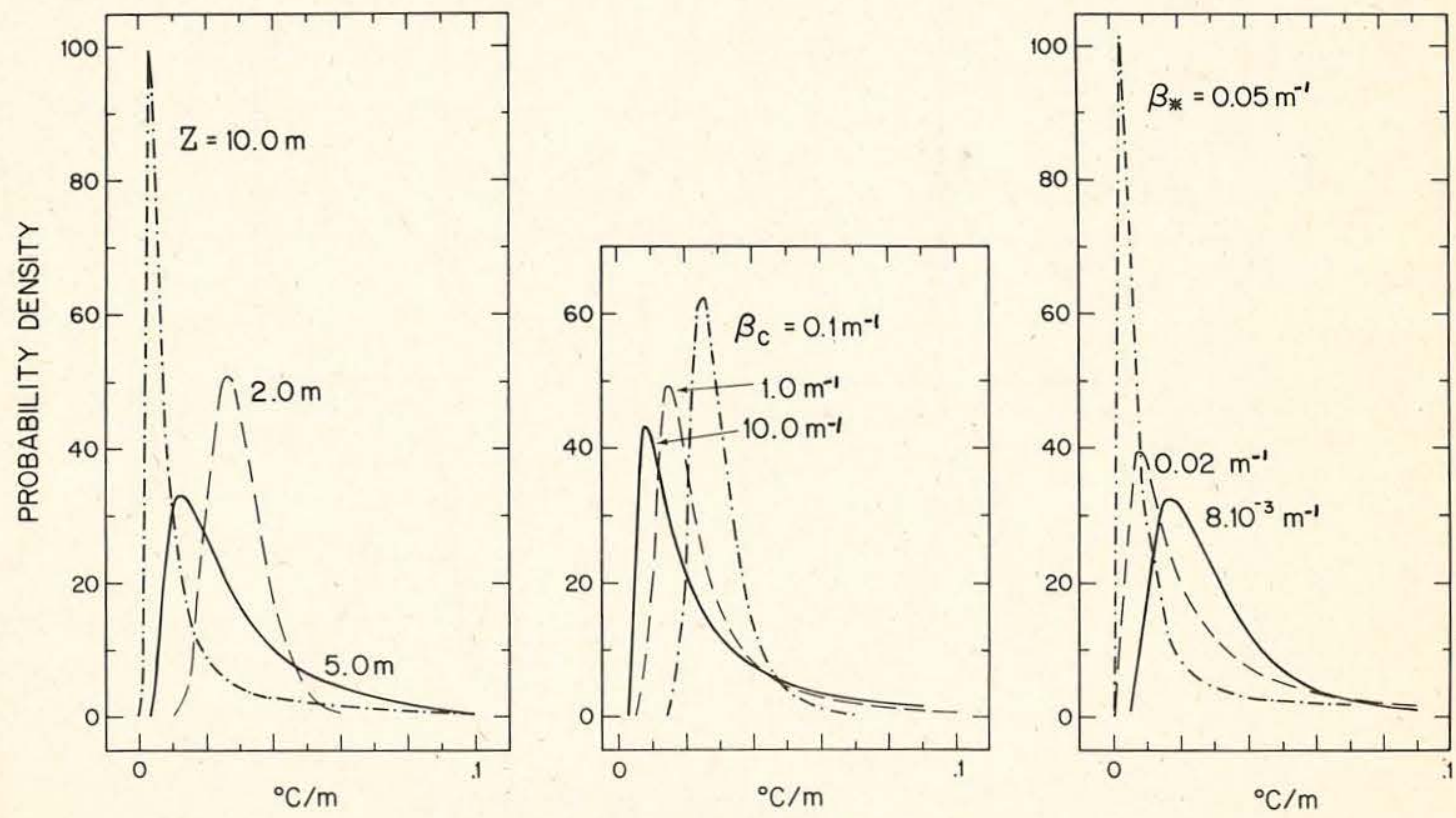

TEMPERATURE GRADIENT

FIG. 10. Sensitivity of the probability density [Eq. (24)] to the various parameters. All curves have $\delta=2 \mathrm{~m}$. Left $\beta_{*}=2 \times 10^{-2} \mathrm{~m}^{-1}, \beta_{c}=\infty$; center $\beta_{*}=2 \times 10^{-2} \mathrm{~m}^{-1}, Z=4 \mathrm{~m}$; right $Z=4 \mathrm{~m}, \beta_{c}=\infty$; other values as indicated. 

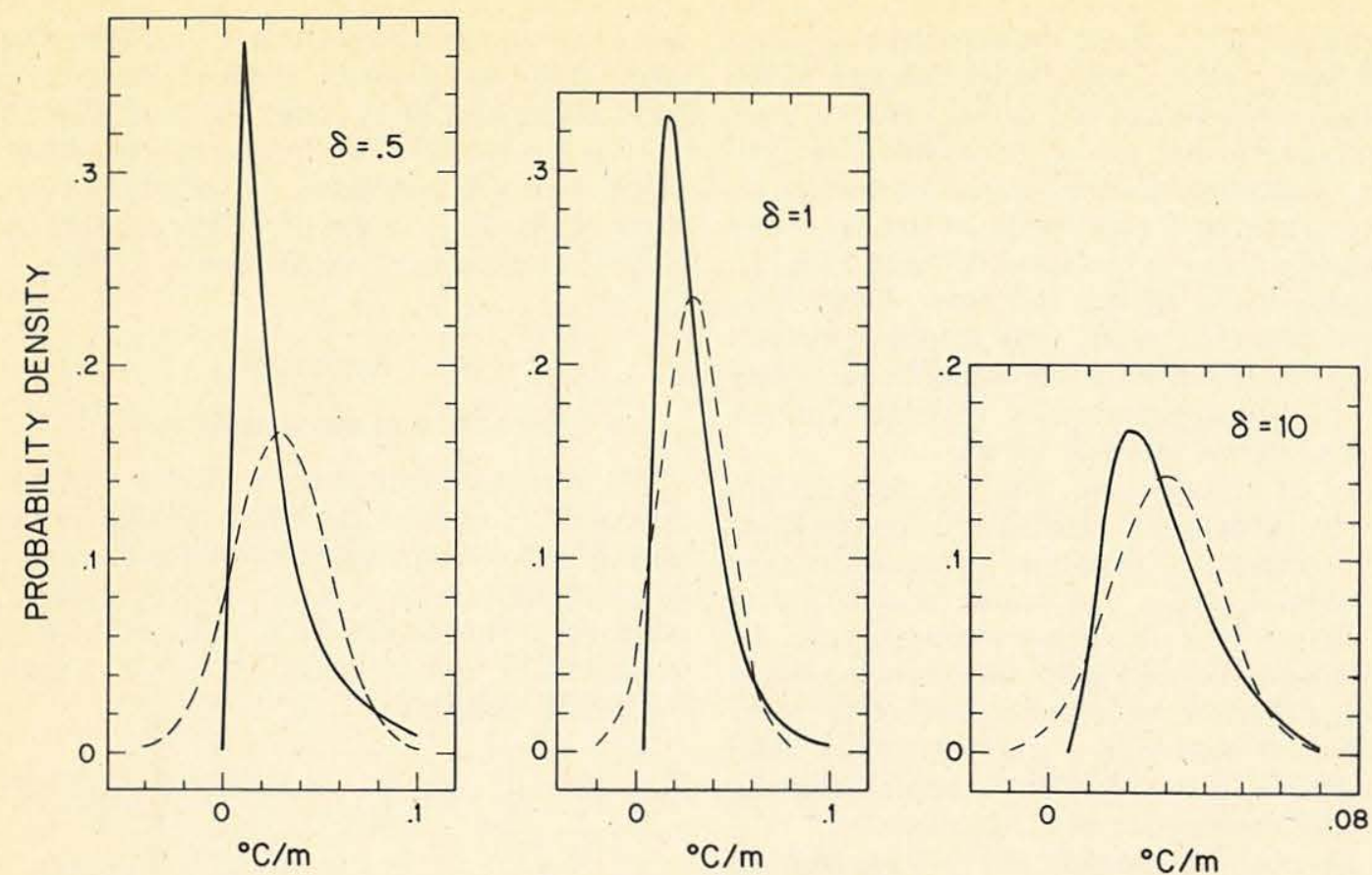

TEMPERATURE GRADIENT

FIG. 11. Comparison of Eq. (24) with the normal distribution (32); parameters as in Table 1.

points, the gradient $g_{i}=\left(T_{i+1}-T_{i}\right) / \delta$. Successive observations are therefore not independent, in contrast to the model which assumes $g$ to be a random variable. Fourth, the joint Gaussian distribution (17), used as a starting point, assigns a finite probability to the event $\eta_{1}>\eta_{2}+y$, i.e., to the possibility that an isotherm $T_{1}$ will overtake another one $T_{2}$, with $T_{1}<T_{2}$, thus creating an inversion. Such a crossing of isotherms should not be allowed however, for it invalidates the notion of a continuous single valued displacement field $\eta(z)$; moreover, the arguments leading to (23) would not hold. Fortunately, for the range of parameters $\delta, Z, \beta_{*}$ considered here the probability $\operatorname{Pr}\left(\eta_{1}>\eta_{2}+y\right)$ turns out to be entirely negligible. Moreover, in the threedimensional problem an inversion can be created without crossing of the isotherms.

A somewhat puzzling point is raised by the use of the spectral representation (26). The mean square strain is given by

$$
\left\langle(d \eta / d z)^{2}\right\rangle=\int_{0}^{\infty} \beta^{2} \phi(\beta) d \beta=\infty .
$$

To avoid this singularity we must require that as $\beta \rightarrow \infty, \phi(\beta) \sim \mathrm{O}\left(\beta^{-n}\right), n>3$; for this reason we originally started this investigation with the convenient representation

$$
\phi(\beta)=K\left(\beta^{2}+\beta_{*}{ }^{2}\right)^{-1}\left(\beta^{2}+\beta_{c}{ }^{2}\right)^{-1},
$$

where the constant $K=2 Z^{2} \pi^{-1} \beta_{*} \beta_{c}\left(\beta_{c}+\beta_{*}\right)$ is such that

$$
\int_{0}^{\infty} \phi(\beta) d \beta=Z^{2}
$$

Here $\beta_{c}$ is a cutoff wavenumber such that for $\beta \ll \beta_{c}$ (33) reduces to (26), while for $\beta \gg \beta_{c}$ the required decaying behavior is obtained. The correlation function corresponding to (33) is simply

$$
\begin{aligned}
\rho(y)=\left[\beta_{c} \exp \left(-\beta_{*} y\right)-\beta_{*} \exp \left(-\beta_{c} y\right)\right] \\
\times\left[\beta_{c}-\beta_{*}\right]^{-1} .
\end{aligned}
$$

If we take for $\beta_{c}$ the value $\beta_{c} \simeq 6.28 \times 10^{-1} \mathrm{~m}^{-1}$ suggested by the observations of Gregg (1977a) the strain variance becomes

$$
\left\langle(d \eta / d z)^{2}\right\rangle=Z^{2} \beta_{c} \beta_{*}=2.57 \times 10^{-1}=(0.5)^{2} .
$$

The curves corresponding to different values of $\beta_{c}$ are shown on Fig. 10, but the best fit to the data (Fig. 9) was obtained for $\beta_{c} \simeq \infty$. This is surprising since a cutoff is clearly required on physical grounds.

\section{Summary and discussion}

We have shown that the temperature structure observed in the ocean is in general the result of two distinct effects: irreversible structures resulting from occasional partial mixing events, and reversible distortions due to the straining by the internal wave field. The observed temperature profiles are quite irregular and cannot be objectively described as a series of well mixed layers separated by high gradient sheets. If the low gradient regions are the re- 
sults of mixing events, these events are rarely complete and they produce only minor changes in the stratification. Over a scale of $5 \mathrm{~m}$, for instance, only $14 \%$ of the profile has gradients smaller than half the mean, indicating that the potential energy increase due to mixing is very small. At smaller scales the mixing effectiveness seems to be larger (Fig. 7), as is suggested also by the run tests. These considerations, however, point only to upper bounds since all the low gradient regions are not necessarily the result of mixing but are also reversible distortions induced by the internal waves.

One way to describe the irregular temperature profiles is by forming the probability density function of the temperature gradients as measured over different vertical scales. For scales from $10 \mathrm{~cm}$ to $10 \mathrm{~m}$ the curves have the same character (Fig. 4). They are highly skewed with the most probable value smaller than the mean and a probability larger than one half of observing a gradient smaller than the mean $\operatorname{Pr}(g<\bar{g})>0.5$. This is reflected in the visual layered appearance of the profiles.

We should note that the data of Fig. 4 are from an area characterized by low levels of mesoscale energy. In other locations there may be a different mix of internal wave strain and irreversible mixing. However, from observations in many areas we believe that the probability density functions we have used are representative of large areas of the ocean thermocline.

The straining of the temperature field by the internal waves produces reversible fluctuations. Previous models interpreted the gradient distributions as being due to the straining by a Gaussian linear internal wave field of a sheet and layer profile. Since there is some evidence that internal waves are nonlinear, and there is none for the sheets and layers, we have taken here the opposite view of a linear temperature profile distorted by nonlinear internal waves. This also results in skewed temperature gradient distributions. The simplified model developed here, which considers only the vertical advection of the isotherms, accounts nonetheless for the distribution of observed gradients for scales larger than about $2 \mathrm{~m}$, but is less convincing at smaller scales. We conclude that over scales larger than $2 \mathrm{~m}$ the nonlinear straining effect could be dominant, but that at the smaller scales both reversible and irreversible structures contribute to the fluctuations in the temperature profile. Because of this interplay between nonlinear strain and partial mixing, it is extremely difficult to trace features in time and space. When attempts at mapping such features are made, it should be kept in mind that straining is significant both in the vertical and horizontal.

Acknowledgments. Conversations with Drs. J. Ehrenberg, L. Goodman and Woollcott Smith were very helpful in the preparation of this paper. The work was supported by ONR (Code 480), Contracts N00014-75-C-0502 to the Applied Physics Laboratory, University of Washington, and N00014-79-C0071 to the Woods Hole Oceanographic Institution. University of Washington, Department of Oceanography, Contribution No. 1211. Woods Hole Oceanographic Institution, Contribution No. 4805.

\section{APPENDIX A}

\section{Derivation of the Density [Eq. (24)]}

We must calculate the probability density function of $T_{2}-T_{1}$ from the joint cumulative distribution (23). We first change variables to simplify the notation: let $z_{2}-z_{1}=\delta, z_{2}{ }^{\prime}-z_{1}{ }^{\prime}=y$; take $z_{1}=0$; since the mean profile $T_{0}(z)=\bar{g} z$ is linear, take $\bar{g}$ $=1$; write $T_{1} \equiv t_{1}=-\eta_{1}, T_{2} \equiv t_{2} \equiv \delta-\eta_{2}$; and rewrite (23) such that

$$
\begin{aligned}
F\left(t_{1}, t_{2}\right)= & \int_{-t_{1}}^{\infty} d \eta_{1} \int_{\delta-t_{2}}^{\infty} d \eta_{2}\left[2 \pi Z^{2}\left(1-\rho^{2}\right)^{1 / 2}\right]^{-1} \\
& \times \exp \left[-\frac{\eta_{1}{ }^{2}+\eta_{2}{ }^{2}-2 \rho \eta_{1} \eta_{2}}{2 Z^{2}\left(1-\rho^{2}\right)}\right],
\end{aligned}
$$

where $\rho=\rho\left(t_{2}-t_{1}\right)=\rho(y)$; we now write the joint density $p\left(t_{1}, t_{2}\right)=f\left(t_{1}, t_{2}\right)=\partial_{t_{1} t_{2}}^{2} F\left(t_{1}, t_{2}\right)$, let $t_{2}-t_{1}$ $=y, t_{2}+t_{1}=2 z($ a new $z)$, then $p(y, z)=f(z-y / 2$, $z+y / 2)$ and the derived density

$$
p(y)=\int_{-\infty}^{\infty} f(z-y / 2, z+y / 2) d z .
$$

Now

$$
\begin{aligned}
& \partial_{t_{1} t_{2}}^{2} F\left(t_{1}, t_{2}\right)=\left[1 / 4 \partial_{z z}^{2}-\partial_{y y}^{2}\right] F(z-y / 2, z+y / 2), \\
& p(y)=\int_{-\infty}^{\infty} d z\left[1 / 4 \partial_{z z}^{2}-\partial_{y y}^{2}\right] F(z-y / 2, z+y / 2) \\
& =1 /\left.4 \partial_{z} F(\cdots, \cdots)\right|_{z=-\infty} ^{z=\infty} \\
& -\int_{-\infty}^{\infty} \partial_{y y}^{2} F(\cdots, \cdots) d z .
\end{aligned}
$$

The first term will turn out to be zero; in the second it will be easiest to calculate

$$
\int_{-\infty}^{\infty} \partial_{y y}^{2} F d z=\partial_{y} \int_{-\infty}^{\infty}\left[\partial_{y} F(z-y / 2, z+y / 2)\right] d z
$$

In (A1), we change variables of integrations according to

$$
\begin{aligned}
\eta_{2}-\eta_{1} & =s(y) \mu, \quad s(y) \equiv Z\{2[1-\rho(y)]\}^{1 / 2} \\
\eta_{2}+\eta_{1} & =2 \sigma(y) \nu \\
\sigma(y) & \equiv Z\{[1+\rho(y)] / 2\}^{1 / 2} \\
d \eta_{1} d \eta_{2} & =Z^{2}\left(1-\rho^{2}\right)^{1 / 2} d \mu d \nu
\end{aligned}
$$

and define $F(z-y / 2, z+y / 2) \equiv G(y, z)$. We have 


$$
\begin{aligned}
& G(y, z)=(2 \pi)^{-1}\left\{\left[\int_{-\infty}^{r} d \mu \int_{b_{2}(\mu)}^{\infty} d \nu\right.\right. \\
&\left.\left.+\int_{r}^{\infty} d \mu \int_{b_{3}(\mu)}^{\infty} d \nu\right] e^{-\left(\nu^{2}+\mu^{2}\right) / 2}\right\},
\end{aligned}
$$

where

$$
\begin{aligned}
r & =r(y) \equiv(\rho-y) / s(y), \\
b_{2}(\mu) & \equiv(-\mu s+2 \delta-2 z-y) /(2 \sigma), \\
b_{3}(\mu) & \equiv(s \mu-2 z+y) /(2 \sigma) .
\end{aligned}
$$

From (A6) it is easy to show that the integrated term in (A3), $\partial_{z} F=\left.\partial_{z} G\right|_{-\infty} ^{\infty}=0$. Following the prescription of (A4) we evaluate

$$
\begin{aligned}
& 2 \pi \partial_{y} G(y, z) \\
& =-\int_{-\infty}^{r} d \mu \frac{\partial b_{2}}{\partial y} \exp \left[-\left(\mu^{2}+b_{2}{ }^{2}\right) / 2\right]-\int_{r}^{\infty} d \mu \frac{\partial b_{3}}{\partial y} \\
& \quad \times \exp \left[-\left(\mu^{2}+b_{3}{ }^{2}\right) / 2\right]+r^{\prime}\left\{\left[\int_{b_{2(r)}}^{\infty} d \nu\right.\right. \\
& \left.\left.-\int_{b_{3(r)}}^{\infty} d \nu\right] \exp \left[-\left(\nu^{2}+r^{2}\right) / 2\right]\right\} .
\end{aligned}
$$

$$
\begin{aligned}
& f(x, y) \\
& g=\Delta T / \delta \\
& \bar{g} \\
& L_{s} \\
& p(\mathbf{x} \mid b)
\end{aligned}
$$

The third term vanishes because $b_{2}(r)=b_{3}(r)$ $=(\rho-2 z) / 2 \sigma ;$ to perform the integration over $(-\infty$ $<z<\infty)$ of the two remaining terms in (A7) we interchange the order of integration: $\int d z d \mu=\int d \mu$ $\times d z$ and change the variable of integration: $-b_{2}(\mu$; $z)=x$ in the first term $-b_{3}(\mu ; z)=x$ in the second,

$$
d z=-\left(\frac{\partial b_{2}}{\partial z}\right)^{-1} d x=-\left(\frac{\partial b_{3}}{\partial z}\right)^{-1} d x=\sigma d x
$$

\section{$T, \bar{T}, T_{0}(z)$}

so that

$$
\begin{aligned}
& -2 \pi \int_{-\infty}^{\infty}\left[\partial_{y} G(y, z)\right] d z \\
& =\int_{-\infty}^{r} e^{-\mu 2 / 2}(1 / 2 \sigma) d \mu \int_{-\infty}^{\infty}\left[-\sigma\left(\mu s^{\prime}+1\right)+2 \sigma^{\prime} \sigma x\right] \\
& \times e^{-x^{2} / 2} d x+\int_{r}^{\infty} e^{-\mu 2 / 2}(1 / 2 \sigma) d \mu \\
& \times \int_{-\infty}^{\infty}\left[\sigma\left(\mu s^{\prime}+1\right)+2 \sigma^{\prime} \sigma x\right] e^{-x^{2} / 2} d x \\
& =-1 / 2(2 \pi)^{1 / 2} \int_{-\infty}^{r}\left(\mu s^{\prime}+1\right) e^{-\mu 2 / 2} d \mu \\
& +1 / 2(2 \pi)^{1 / 2} \int_{r}^{\infty}\left(\mu s^{\prime}+1\right) e^{-\mu 2 / 2} d \mu \\
& y=z_{2}{ }^{\prime}-z_{1}{ }^{\prime} \\
& z_{*}, z_{2}, z_{1} \\
& z^{\prime}, z_{2}{ }^{\prime}, z_{1}{ }^{\prime} \\
& \begin{array}{l}
Z \\
\beta, \beta_{*}
\end{array} \\
& \delta \\
& \underset{\eta}{\zeta}=\left(\xi_{1}, \xi_{2}\right)
\end{aligned}
$$

Finally,

$$
-(2 \pi)^{1 / 2} \partial_{y} \int_{-\infty}^{\infty}\left(\partial_{y} G\right) d z=-e^{-r^{2} / 2}\left[r^{\prime}\left(r s^{\prime}+1\right)-s^{\prime \prime}\right] .
$$

Substituting these results into (A3) gives

\section{$\operatorname{Pr}(e)$}

$s(y)$ $=Z\{2[1-\rho(y)]\}^{1 / 2}$

$$
\begin{aligned}
& \mu \\
& \xi
\end{aligned}
$$

\section{APPENDIX B}

\section{Notation}

a function of $x$ and $y$ observed gradient mean gradient

typical sheet thickness in a sheet and layer model probability density function of the random variables $\mathbf{x}=x_{1} \cdots x_{n}$ with $b$ as a given parameter probability of the event $e$ position vector

rms value of the displacement difference

temperature, mean temperature at a point, equilibrium profile in the absence of internal waves

vertical separation of equilibrium depths of two isotherms

depth in general observation depth equilibrium depth of an isotherm

rms vertical displacement vertical wavenumber, bandwidth

vertical interval over which the temperature difference $\Delta T$ is observed and the gradient computed

a six-vector $\left(\xi_{1}, \xi_{2}\right)$

vertical displacement of isotherm from its equilibrium position

average number of sheets per meter

displacement vector of a particle 
$\rho=\rho(y)=\left\langle\eta_{2} \eta_{1}\right\rangle / Z^{2}$ correlation function of vertical displacements

$\phi$ power spectral density.

\section{REFERENCES}

Bendat, J. S., and A. G. Piersol, 1971: Random Data: Analysis and Measurement Procedures. Wiley-Interscience, $407 \mathrm{pp}$.

Briscoe, M. G., 1977: Gaussianity of internal waves. J. Geophys. Res., 82, 2117-2126.

Cooper, J. W., and H. Stommel, 1968: Regularly spaced steps in the main thermocline near Bermuda. J. Geophys. Res., $73,5849-5854$.

Cox, R. D., and P. H. Lewis, 1966: The Statistical Analysis of Series of Events. Methuen, $285 \mathrm{pp}$.

Desaubies, Y. J. F., 1976: Analytical representation of internal wave spectra. J. Phys. Oceanogr., 6, 976-981.

- 1978: On the scattering of sound by internal waves in the ocean. J. Acoust. Soc. Amer., 64, 1460-1469.

Eckart, C., 1948: An analysis of the stirring and mixing of incompressible fluids. J. Marine Res., 7, 265-275.

Eriksen, C. C., 1978: Measurements and models of finestructure, internal gravity waves and wave breaking in the deep ocean. J. Geophys. Res., 83, 2989-3009.

Ewart, T. E., 1980: A numerical simulation of the effects of oceanic finestructure on acoustic transmission. J. Acoust. Soc. Amer., 67, 496-503.

Flatté, S. M., R. Leung and S. Y. Lee, 1980: Frequency spectra of acoustic fluctuations caused by oceanic internal waves and other finestructure. J. Acoust. Soc. Amer., 68, 1773-1779.

Garrett, C., 1979: Mixing in the ocean interior. Dyn. Atmos. Oceans (in press).

, and W. Munk, 1971: Internal wave spectra in the presence of finestructure. J. Phys. Oceanogr., 1, 196-202.
— , and _ 1975: Space-time scales of internal waves: A progress report. J. Geophys. Res., 80, 291-297.

Gregg, M. C., 1977a: A comparison of finestructure spectra from the main thermocline. J. Phys. Oceanogr., 7, 33-40.

_ 1977b: Variations in the intensity of small-scale mixing in the main thermocline. J. Phys. Oceanogr., 7, 436-454.

- 1980: Microstructure patches in the thermocline. J. Phys. Oceanogr., 10, 915-943.

_ , and T. B. Sanford, 1980: Signatures of mixing from the Bermuda Slope, the Sargasso Sea, and the Gulf Stream. J. Phys. Oceanogr., 10, 105-127.

Hayes, S. P., T. M. Joyce and R. C. Millard, 1975: Measurements of vertical finestructure in the Sargasso Sea. $J$. Geophys. Res., 80, 314-320.

Holloway, G., 1980: Oceanic internal waves are not weak waves. J. Phys. Oceanogr., 10, 906-914.

Johnson, C. L., C. S. Cox and B. Gallagher, 1978: The separation of wave induced and intrusive oceanic finestructure. J. Phys. Oceanogr., 8, 846-860.

Joyce, T. M., and Y. J. F. Desaubies, 1977: Discrimination between internal waves and temperature finestructure. J. Phys. Oceanogr., 7, 22-32.

Levine, M. D., 1979: Statistical description of internal waves and temperature finestructure in the deep ocean. Ph.D. dissertation, University of Washington, $173 \mathrm{pp}$.

McKean, R. S., 1974: Interpretation of internal wave measurements in the presence of finestructure. J. Phys. Oceanogr., 4, 200-213.

Phillips, O. M., 1971: On spectra measured in an undulating layered medium. J. Phys. Oceanogr., 1, 1-6.

Reid, R. O., 1971: A special case of Phillips' general theory of sampling statistics for a layered medium.J.Phys. Oceanogr. 1, 61-62.

Woods, J. D., 1968: Wave-induced shear instability in the summer thermocline. J. Fluid Mech., 32, 791-800. 
MANDATORY DISTRIBUTION LIST

FOR UNCLASSIFIED TECHNICAL REPORTS, REPRINTS, AND FINAL REPORTS

PUBLISHED BY OCEANOGRAPHIC CONTRACTORS

OF THE OCEAN SCIENCE AND TECHNOLOGY DIVISION

OF THE OFFICE OF NAVAL RESEARCH

(REVISED NOVEMBER 1978)

1 Deputy Under Secretary of Defense

(Research and Advanced Technology)

Military Assistant for Environmental Science

Room 30129

Washington, D.C. 20301

Office of Naval Research

800 North Quincy Street

Arlington, VA 22217

3 ATTN: Code 483

1 ATTN: Code 460

2 ATTN: 102B

1 CDR Joe Spigai, (USN)

ONR Representative

Woods Hole Oceanographic Inst.

Woods Hole, MA 02543

Commanding officer

Naval Research Laboratory

Washington, D.C. 20375

6 ATTN: Library, Code 2627

12 Defense Technical Information Center

Cameron Station

Alexandria, VA 22314

ATTN: DCA

Commander

Naval Oceanographic Office

NSTL Station

Bay St. Louis, MS 39522

1 ATTN: Code 8100

1 ATTN: Code 6000

1 ATTN: Code 3300

1 NODC/NOAA

Code D781

Wiscons in Avenue, N.W.

Washington, D.C. 20235

1 Mr. Michael H. Kelly

Administrative Contracting Officer

Department of the Navy

Office of Naval Research

Eastern/Central Regional Office

Building 114, Section D

666 Summer Street

Boston, MA 02210 
UNCLASSIFIED $9 / 8]$

SECURITY CLASSIFICATION OF THIS PAGE (When Data Entored)

\begin{tabular}{|c|c|}
\hline REPORT DOCUMENTATION PAGE & $\begin{array}{c}\text { READ INSTRUCTIONS } \\
\text { BEFORE COMPLETING FORM }\end{array}$ \\
\hline \begin{tabular}{l|l|} 
1. PEPORT NUMBER & 2. GOVT ACCESSION NO. \\
WHOI - $81-83$ &
\end{tabular} & 3. RECIPIENT'S CATALOG NUMBER \\
\hline \multirow[t]{2}{*}{$\begin{array}{l}\text { 4. TITLE (and Subtita) } \\
\text { REVERSIBLE AND IRREVERSIBLE FINESTRUCTURE }\end{array}$} & $\begin{array}{l}\text { 5. TYPE OF REPORT A PERIOD COVERED } \\
\text { Technical }\end{array}$ \\
\hline & $\begin{array}{l}\text { 6. PERFORMING ORG. REPORT NUMBER } \\
\text { WHOI contr. } 4805\end{array}$ \\
\hline $\begin{array}{l}\text { 7. AUTHOR(s) } \\
\text { Yves Desaubies and M.C. Gregg }\end{array}$ & $\begin{array}{l}\text { 8. CONTRACT OR GRANT NUMBER(a) } \\
\text { N00014-75-C-0502 } \\
\text { N00014-79-C-0071 }\end{array}$ \\
\hline $\begin{array}{l}\text { 9. PERFORMING ORGANIZATION NAME AND ADDRESS } \\
\text { Woods Hole Oceanographic Institution } \\
\text { Woods Hole, Massachusetts } 02543\end{array}$ & $\begin{array}{l}\text { 10. PROGRAM ELEMENT, PROJECT, TASK } \\
\text { AREA \& WORK UNIT NUMBERS }\end{array}$ \\
\hline $\begin{array}{l}\text { 11. CONTROLLING OFFICE NAME AND ADDRESS } \\
\text { NORDA/National Space Technology Laboratory } \\
\text { Bay St. Louis, MS } 39529\end{array}$ & $\begin{array}{l}\text { 12. REPORT DATE } \\
\text { September } 1987 \\
\text { 13. NUMBER OF PAGES }\end{array}$ \\
\hline 14. MONITORING AGENCY NAME \& ADDRESS(il different from Controlline Office) & $\begin{array}{l}\text { 15. SECURITY CLASS. (of this roport) } \\
\text { UnClaSSified } \\
\text { 15a. DECLASSIFICATION/DOWNGRADING } \\
\text { SCHEDULE }\end{array}$ \\
\hline
\end{tabular}

16. DISTRIBUTION STATEMENT (of this Roport)

Approved for public release; distribution unlimited.

17. DISTRIBUTION STATEMENT (of the abstract ontered in Block 20, If different from Report)

18. SUPPLEMENTARY NOTES

Reprinted from: Journal of Physical Oceanography 11(4): 541-546 April 1981).

19. KEY WORDS (Continue on reverae eide if neceseary and Identify by block number)

1. Internal waves

2. Finestructure

3. Mixing

20. ABSTRACT (Continue on roverso ofde if necoseary and identify by block number)

Various statistics of temperature profiles are examined in an attempt to distinguish irreversible structures due to mixing from reversible distortions induced by internal wave straining. Even if all the low gradient regions were the result of mixing events, an analysis of the profiles shows that such events are rare and most often incomplete. An upper bound on the mixing effectiveness is obtained; it increases as the vertical scale decreases. Taking next the opposite view that internal wave straining is the 


\section{0. cont.}

sole process, an analytic model is developed to calculate the probability density function of temperature gradients. The model considers the straining by a weakly nonlinear Gaussian internal wave field of a linear temperature profile. The nonlinearity of the field is essential to account for the skewness of the probability distributions. Comparisons with data are quite satisfactory at scales larger than $\sim 2 \mathrm{~m}$, less so at smaller scales. We conclude that nonlinear effects are important; at scales larger than $\sim 2 \mathrm{~m}$ straining is dominant with very little mixing, while at smaller scales irreversible structures are more prevalent. 


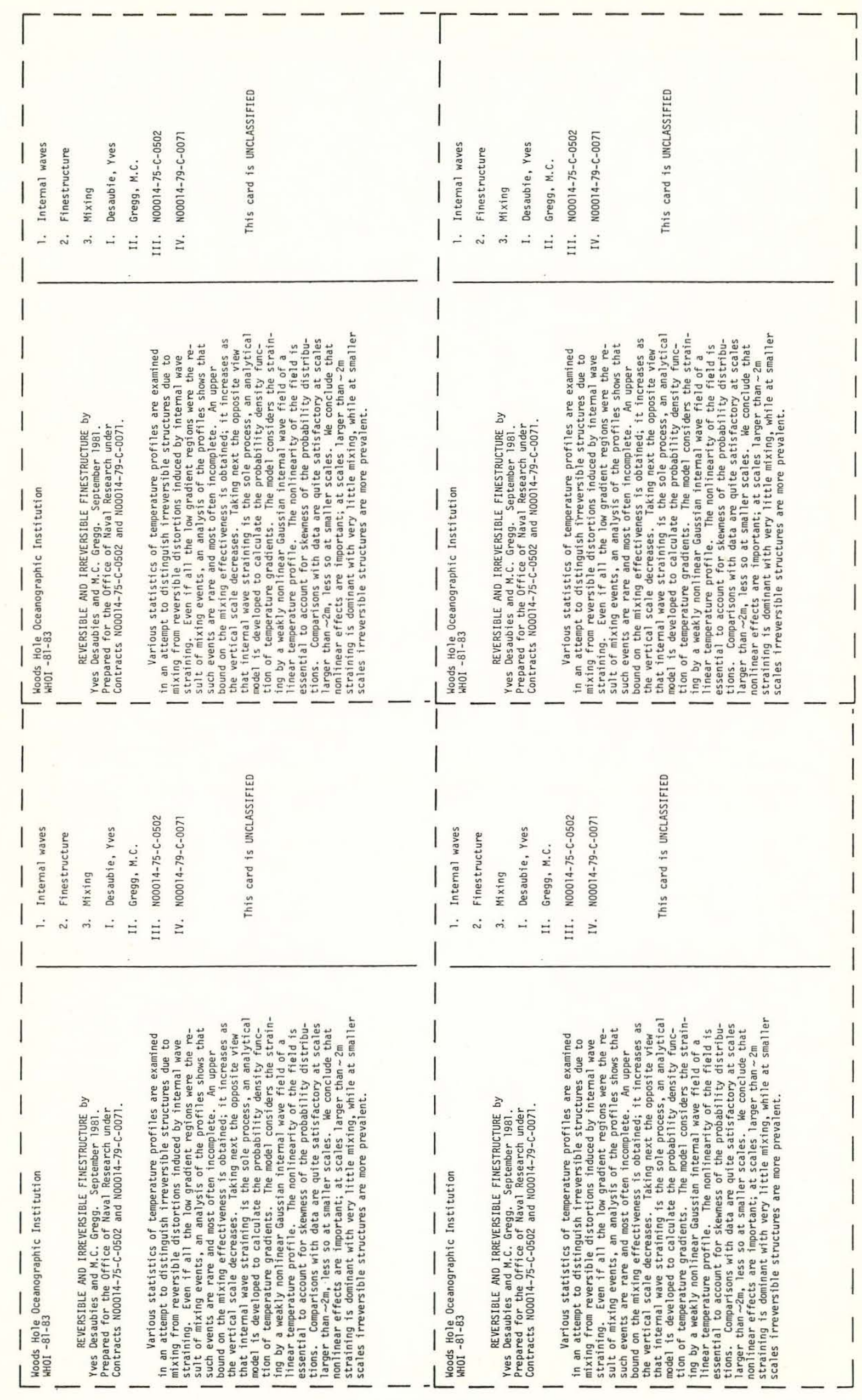

